\title{
eNeuro
}

Research Article: New Research / Sensory and Motor Systems

\section{Sensorimotor learning in response to errors in task performance}

https://doi.org/10.1523/ENEURO.0371-21.2022

Cite as: eNeuro 2022; 10.1523/ENEURO.0371-21.2022

Received: 13 September 2021

Revised: 18 January 2022

Accepted: 23 January 2022

This Early Release article has been peer-reviewed and accepted, but has not been through the composition and copyediting processes. The final version may differ slightly in style or formatting and will contain links to any extended data.

Alerts: Sign up at www.eneuro.org/alerts to receive customized email alerts when the fully formatted version of this article is published.

Copyright (c) 2022 Sadaphal et al.

This is an open-access article distributed under the terms of the Creative Commons Attribution 4.0 International license, which permits unrestricted use, distribution and reproduction in any medium provided that the original work is properly attributed. 
Sensorimotor learning in response to errors in task performance.

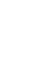

Dhwani P. Sadaphal ${ }^{1}$, Adarsh Kumar ${ }^{1,2}$ and Pratik K. Mutha ${ }^{1,3}$

${ }^{1}$ Center for Cognitive and Brain Sciences, ${ }^{2}$ Department of Mechanical Engineering,

6 and ${ }^{3}$ Department of Biological Engineering, Indian Institute of Technology

7 Gandhinagar, India.

9 Abbreviated Title: Strategic compensation of task errors

Author Contributions: DPS, AK and PKM designed the study, DPS collected the

data, DPS, AK and PKM authors analyzed the data, DPS and PKM wrote the paper.

\section{Correspondence should be addressed to:}

Pratik K. Mutha

Block 5, Room 316A

Indian Institute of Technology Gandhinagar

Palaj, Gandhinagar - 382355

Gujarat, India

Email: pm@iitgn.ac.in

Number of Figures: 5

Number of Tables: 0

Number of Multimedia: 0

Number of words for Abstract: 209

Number of words for Significance Statement: 120

\section{Number of words for Introduction: 750}

Number of words for Discussion: 1721

Acknowledgements: We thank Gaurav Panthi for helpful discussions.

Conflict of Interest: Authors report no conflict of interest

1 Funding sources: This work was partially supported by grants from the Department of Science and Technology, Government of India to PKM. We also acknowledge 


\section{ABSTRACT}

36

37 The human sensorimotor system is sensitive to both limb-related prediction errors

38 and task-related performance errors. Prediction error signals are believed to drive

39 implicit refinements to motor plans. However, an understanding of the mechanisms

40 that performance errors stimulate has remained unclear largely because their effects

41 have not been probed in isolation from prediction errors. Diverging from past work,

42 we induced performance errors independent of prediction errors by shifting the

43 location of a reach target but keeping the intended and actual kinematic

44 consequences of the motion matched. Our first two experiments revealed that rather

45 than implicit learning, motor adjustments in response to performance errors reflect

46 the use of deliberative, volitional strategies. Our third experiment revealed a potential

47 dissociation of performance-error-driven strategies based on error size. Specifically,

48 behavioral changes following large errors were consistent with goal-directed or

49 model-based control, known to be supported by connections between prefrontal

50 cortex and associative striatum. In contrast, motor changes following smaller

51 performance errors carried signatures of model-free stimulus-response learning, of

52 the kind underpinned by pathways between motor cortical areas and sensorimotor

53 striatum. Across all experiments, we also found remarkably faster re-learning,

54 advocating that such "savings" is associated with retrieval of previously learned

55 strategic error compensation and may not require a history of exposure to limb-

56 related errors. 


\section{SIGNIFICANCE STATEMENT}

58

59 Humans adjust their actions if they do not produce desired limb-related sensory

60 consequences or task-related outcomes. We probed whether task-related

61 performance errors induce implicit changes to motor plans at all, or simply trigger the

62 deliberate selection of different actions. We induced performance errors in isolation,

63 and found that they were compensated entirely via intentional, strategic mechanisms

64 consistent with improved action selection. Strategies also appeared to be sensitive to

65 error size, and transitioned from stimulus-response associative behavior to goal-

66 directed control as error magnitude increased. Across all experiments, we also found

67 faster re-learning or "savings", substantiating the view that savings is associated with

68 strategy-use, and does not depend on experience of limb-related prediction errors

69 that bring about implicit adjustments to action plans. 
72 Studies of motor adaptation, the capacity to recalibrate our actions to changing body

73 and environmental conditions, have been instrumental in characterizing many

74 fundamental principles of sensorimotor learning. Adaptation paradigms have typically

75 employed different visual (Morehead et al., 2017; Scheidt et al., 2005) or dynamic

76 (Lefumat et al., 2015; Sainburg et al., 1999; Shadmehr and Mussa-Ivaldi, 1994)

77 perturbations that produce discrepancies in the actual versus expected limb-related

78 sensory feedback. It is generally believed that such sensory prediction errors (SPEs) are compensated by implicitly recalibrating motor plans (Mazzoni and Krakauer, 2006; Morehead et al., 2017; Oza et al., 2020). SPE-driven changes in motor output are dependent on cerebellar (Flament et al., 1996; Martin et al., 1996; Morehead et al., 2017) and posterior parietal networks (Clower et al., 1996; Della-Maggiore et al., 2004; Kumar et al., 2020); disruption in these regions, either naturally due to Stroke or degeneration, or artificially using brain stimulation techniques, produces clear deficits in SPE-based learning.

Perturbations applied to moving effectors produce not just SPEs, but can also result in task performance errors (TPEs). In goal-directed motion, TPEs could arise from a failure to achieve the movement goal (missing a spatial target, for instance), or when a target moves to a different location while the action is being performed. Learning to compensate TPEs plausibly requires intact cortico-striatal circuits (Anguera et al., 2010; Taylor and Ivry, 2012), although a measure of the TPE itself could come from the simple spike discharge of cerebellar Purkinje neurons (Popa et al., 2017). However, a clear understanding of the computational and psychological mechanisms that drive changes in motor behavior upon exposure to recurring TPEs, has remained elusive. While early work hinted that TPEs may not induce an implicit adaptive response, it did not elaborate on the algorithms employed (Diedrichsen et al., 2005). Later studies suggested that TPEs could provoke use of deliberative movement re-aiming strategies (McDougle and Taylor, 2019; Taylor et al., 2014), but an alternative proposition has been put forth in more recent work. This latter set of studies, which have probed the influence of binary TPEs on learning, suggests that like SPEs, TPEs can drive implicit learning, and net adaptation reflects the sum of two implicit processes, one driven by SPE and the other by TPE (Kim et al., 2019; 
Leow et al., 2018; Van der Kooij et al., 2018). These two views thus differ in terms of how TPEs contribute: one suggests that they drive the formulation of an explicit strategy, while the other invokes implicit recalibration.

This debate arises primarily because TPEs have rarely been elicited independent of SPEs. When these errors co-occur, it is likely that they interact, which, neuroanatomically, could be facilitated via connections between the basal ganglia and the cerebellum (Bostan and Strick, 2018). Furthermore, this interaction may be competitive, with SPEs dominating the adaptative response (Wang et al., 2019). This is supported by findings in healthy individuals who adapt to SPEs even if it amplifies TPEs (Mazzoni and Krakauer, 2006), or who cannot correct for TPEs due to task constraints (Tseng et al., 2007). Likewise, Stroke patients with lesions circumscribed to right inferior frontal cortex show complete adaptation to SPEs despite failing to correct for TPEs (Mutha et al., 2011). Given this overwhelming influence of SPEs when imposed concurrently with TPEs, it is perhaps not surprising that mechanisms through which TPEs alone are compensated have remained unclear.

Resolving the mechanisms underlying TPE-mediated changes in motor behavior also has implications for understanding the formation of long-term motor memories. Such latent memories enable faster learning upon re-exposure to the perturbation, a phenomenon termed "savings". While there is evidence that savings is promoted via strategic re-aiming (Haith et al., 2015; Huberdeau et al., 2015; Morehead et al., 2015), some studies have linked it to other processes including implicit mechanisms (Coltman et al., 2019; Yin and Wei, 2020), action repetition (Huang et al., 2011) and a memory of the experienced errors that in turn modulates error sensitivity (Herzfeld et al., 2014). Based on these diverse results, one cannot be certain whether it is improved action selection (mediated by TPEs) or improved action execution (mediated by SPEs) or a combination of the two that contributes to long-term motor memory formation that facilitates savings.

Here we examined how humans learn to compensate consistent TPEs imposed in isolation from SPEs, and if they express as savings the acquired memory when reexposed to the learning environment. We also probed whether and how the magnitude of the TPE influences the ensuing changes in motor output. 


\section{MATERIALS AND METHODS}

140

\section{Subjects}

142 We recruited 76 healthy, right-handed individuals between the age 18 and 30 years

143 across 3 different experiments. Handedness was assessed using the Edinburgh

144 Handedness Inventory. All subjects were naïve to the expected outcomes of the

145 experiment, provided written informed consent before participating, and were paid for

146 their time. The study was approved by our Institute Ethics Committee. One subject

147 was excluded (see below), resulting in a total of 75 subjects (mean age $=22.64 \pm 0.34$ years, 27 females) whose data were analyzed.

\section{Experimental setup}

151 Subjects sat on a height-adjustable chair facing a large, horizontally placed digitizing

152 tablet and used a hand-held stylus to make planar, targeted reaching movements on

153 it (Figure 1A). All movements were made with the right hand. Subjects received

154 visual feedback of their hand (stylus) position on a mirror that reflected a high-

155 definition display placed directly above it. The mirror was aligned parallel to the

156 screen and the digitizing tablet, and prevented direct view of the moving limb. Hand

157 position was displayed as a circular cursor $(0.5 \mathrm{~cm}$ diameter) along with a circular

158 start position ( $1 \mathrm{~cm}$ diameter) and targets $(1.5 \mathrm{~cm}$ diameter) for the reach.

To begin a trial, subjects first brought the cursor into the start circle. After a delay of $500 \mathrm{~ms}$, a target appeared at one of four locations $\left(45^{\circ}, 135^{\circ}, 225^{\circ}\right.$ or $\left.315^{\circ}\right)$ along with an audio beep that indicated to subjects that they should start moving. Across all experiments, the distance between the start position and the target was fixed at $10 \mathrm{~cm}$, and subjects were encouraged to move as quickly as possible, but no specific constraints were imposed on either reaction time or movement time. Further, cursor feedback was provided during the entire reach and was always veridical with the actual position of the hand. 


\section{Experimental blocks}

172 In all 3 experiments of this study, subjects performed 4 blocks of trials: baseline,

173 learning, washout and savings. Baseline trials comprised of reaches to fixed,

174 stationary targets. This was followed by the learning block in which the target

175 location was shifted, or "jumped", counterclockwise on each trial. The shift was

176 achieved by extinguishing the originally displayed target ("original" target) and

177

178

179

180 immediately displaying a new one ("new" target). The magnitude of the target-shift was $45^{\circ}$ in experiments 1 and 2 , while it was $15^{\circ}, 30^{\circ}$ or $60^{\circ}$ for the different groups of experiment 3 (Figures 1B and 1D, also see below). The shift was initiated as soon as subjects breached the start circle boundary (moved $3 \mathrm{~mm}$ from the center of the start circle), and enabled us to impose a TPE. The learning block, in which subjects learned to predictively account for the TPE (Figure 1C), was followed by washout trials that were similar to baseline in that there was no target-shift, and the original target remained on the screen for the entire trial. After washout, we probed for "savings" by exposing subjects to target-shifts as in the earlier learning block. Specific task instructions were given prior to the onset of each block (see below).

To gain some familiarity with the setup and the task display, subjects first performed 10 no-shift trials and then 2 target-shift trials; these 12 practice trials were not analyzed. Before they attempted the no-shift practice trials, subjects were explained what they would see on the screen and told that they should reach from the start circle to the target. Prior to the practice target-shift trials, they were told that they might experience trials in the task where the target would jump to a different location. They then performed 2 such trials as practice. Throughout the experiment including practice trials, at the end of each trial, subjects were given points $(10,5,3$ or none) depending on the accuracy of their movement. Accuracy was calculated relative to the original target on baseline and washout trials, and the new target for the learning and savings trials. Points were not analyzed.

\section{Experiment 1}

In our first experiment $(n=30)$, subjects performed 56 baseline, 112 learning, 112 washout and 112 savings trials. As described earlier, baseline and washout trials comprised of reaches to stationary targets, while the target was jumped $45^{\circ}$ counterclockwise during the learning and savings blocks. Additionally, interspersed 
within the learning and savings blocks were 3 sub-blocks of 4 trials each on which the target was not shifted; these trials were thus similar to baseline (Figure 1B) and did not induce a TPE. Each of the no-shift sub-blocks occurred after every 28 targetshift trials. In all, subjects performed 416 trials in this first experiment.

Subjects were given verbal instructions before each of the main experimental blocks and also before each no-shift sub-block embedded within the learning and savings blocks. Prior to the baseline block, subjects were told to reach to the target that would be displayed on the screen, and were also informed that its position would not change. Following baseline and prior to the onset of the learning block, subjects were told that the target would now start "jumping", and that they should reach to the new target. Further, before each no-shift sub-block, they were told that the target would now stop jumping and they should move to the original target. Similarly, at the end of each no-shift sub-block, subjects were informed that the target would start jumping again and they should go to the new target. Instructions before the washout block were similar to those given before the no-shift sub-blocks. Instructions provided before the savings block were the same as those given prior to the learning block. In sum, verbal instructions were given every time the target-shift conditions were about to change.

\section{Experiment 2}

The design of our second experiment $(n=10)$ was motivated by the work of Taylor et al. (2014), who used verbal reports of subjects' intended aiming direction to estimate their use of cognitive strategies. The setup and general task environment remained similar to that of experiment 1 . Subjects performed 40 baseline, 112 learning, 40 washout and 112 savings trials. The reach target remained stationary on the baseline and washout trials, while $45^{\circ}$ counterclockwise target jumps were introduced on each trial of the learning and savings blocks (Figure 1D). Target presentation and timing of the jump remained similar to experiment 1 . The no-shift sub-blocks were not employed in this experiment.

In addition to the start circle and the target, a ring of 72 numerical landmarks (numbered from 0 to 71 , increasing counterclockwise) placed at $5^{\circ}$ intervals along the periphery of a virtual circle of $10 \mathrm{~cm}$ diameter (corresponding to the target 
distance) was also presented on each trial of all 4 blocks (Figure 1D). Since the target could appear at any one of four different locations, the ring was rotated such that landmark "0" always coincided with the location of the original target for that trial while landmark "9" always corresponded to the location of the new target displayed on learning and savings trials ( $45^{\circ}$ counterclockwise). The ring was presented simultaneous with the original target and it disappeared once the subjects crossed the edge of the start circle. Importantly, on every trial, before they initiated their movement, subjects were required to verbally report their aiming direction by stating the approximate numerical landmark they intended to move to. This number was recorded by the experimenter.

As in experiment 1, subjects were also informed about target behavior prior to each block. Briefly, before baseline trials, subjects were told that they should move to the target that would be displayed on the screen, and that its location would not change during the trial. Prior to the learning block, subjects were informed that the target would now start "jumping" during the trial and they should reach to the new target. Before washout, they were again informed that the target would stop jumping and they should move to the original target. Finally, before the savings block, they were told that the targets would start jumping again and they should go to the new target.

\section{Experiment 3}

In Experiment 3 ( $n=36$, one subject was excluded from the analysis, so final $n=$ 35), we aimed to understand the influence of TPE magnitude on changes in motor behavior. Subjects were assigned to three different groups, that differed in terms of the magnitude of the target-shift experienced $\left[15^{\circ}(n=11), 30^{\circ}(n=12)\right.$, or $60^{\circ}(n=$ 12)]. All jumps were counterclockwise as before, and all other aspects of this experiment were identical to experiment 1 (Figure 1B). Thus, subjects performed 4 blocks: baseline (56 trials), learning (112 trials), washout (112 trials) and savings (112 trials). Targets remained stationary during the baseline and washout blocks, while they were shifted on learning and savings trials. Three no-shift sub-blocks (4 trials each) were also embedded within the learning and savings blocks. Instructions to subjects and their schedule remained the same as in experiment 1. 


\section{Data Analysis}

\section{Variables}

275 Data were analyzed using custom Matlab scripts. Hand $X$ and $Y$ position data were

276 filtered using a low-pass Butterworth filter with $10 \mathrm{~Hz}$ cutoff. Position data were

277 differentiated to obtain the speed profile. Movement onset was defined as the point

278 at which hand speed first crossed $5 \%$ of maximum movement speed. Reaction time

$279(\mathrm{RT})$, a variable of interest in experiments 1 and 3, was calculated as the difference 280 between the time of movement onset and the time of target presentation. Our other 281 key measure was the deviation in hand movement direction relative to the direction 282 of the original target. This was calculated as the angle between two lines: the line 283 joining the center of the start circle and the original target, and the line joining the 284 center of the start circle and the hand position at peak speed. On a few trials, more 285 than one peak could occur. For example, on the early learning trials (Figure 1C), 286 subjects could make an initial outward movement to the original target and then correct it online to go to the new target, resulting in two peaks in the speed profile. In such cases, hand position at the first large $(>15 \mathrm{~cm} / \mathrm{s})$ peak, corresponding to the outward movement to the original target, was chosen for the calculation of hand deviation since this would serve as a more appropriate indicator of the subjects' initial movement plan. Counterclockwise and clockwise deviations relative to the original target were treated as positive and negative respectively.

\section{Outlier removal}

Firstly, trials on which subjects did not move, or moved but lifted the stylus off the digitizing tablet leading to data loss, were marked as bad trials. Second, outliers were identified based on the hand deviation data. For the baseline and washout blocks, we first calculated the mean hand deviation across all trials of that block, and then labeled as an outlier any trial on which the hand deviation was more than \pm 3 standard deviations from the corresponding mean. For the learning and savings blocks, outliers were marked as those trials on which the hand deviation was more than \pm 3 times the magnitude of the target-shift. Following this procedure, one subject

303 from the $15^{\circ}$ jump group of experiment 3 ended up with $136 \mathrm{bad} /$ outlier trials (out of 304416 trials performed); this subject was excluded entirely. Across all the remaining 75 305 subjects, $1.34 \%$ of the trials were labeled as bad trials or outliers and removed from 306 the analysis. 
308 Further analyses and statistics:

309 Following outlier removal, potential baseline biases in reach direction were

310 eliminated by subtracting the mean baseline hand deviation from the hand deviation

311 on each trial; these baseline-subtracted values were used for further analyses.

312 Average hand deviation and RT on the last twelve baseline trials were taken as an

313 indicator of late baseline behavior. We also computed the mean hand deviation and

314 RT on the first and last unique reaches to each target (four trials) of the learning, washout and savings blocks. This provided a measure of early and late-stage performance in each of these blocks. Performance on the no-shift sub-blocks was assessed by averaging hand deviation and RT across all four trials of each sub-

318 block.

319

320

We typically used parametric tests (analysis of variance (ANOVA) or t-tests) to compare across different stages or groups after checking the underlying assumptions. Wilcoxon signed rank tests were used in place of t-tests if the data were found to deviate from normality (assessed via Shapiro-Wilk tests). Levene's test was used to assess homogeneity of variance required for ANOVA. If this was violated, Welch's ANOVA was used. Sphericity violations in repeated measures ANOVAs were accounted for via Greenhouse-Geisser corrections. Cohen's d, matched ranked biserial correlation and $\omega^{2}$ were used as measures of effect size for the t-test, Wilcoxon signed rank test and ANOVA respectively. The significance level was set at $p=0.05$ for all tests. Further, given the known issues with RT distributions (Wagenmakers and Brown, 2007), RT comparisons were also made using estimation statistics, which focus on the effect size and its precision. Bayesian

332 inference methods were also used when warranted. Statistical analyses were carried

333 out using $\mathrm{R}$ (version 4.0.0) and JASP (version 0.13.1).

\section{RESULTS}

336

In experiment 1 , subjects reached to 1 of 4 visual targets under veridical feedback

338 provided by means of a cursor representing hand position (Figure 1A). On learning

339 trials, the target was "jumped" counterclockwise by $45^{\circ}$, thereby inducing a TPE 
340 (Figure 1B). Subjects were informed about the occurrence of the target-shift and 341 instructed to reach to the new target. Interspersed within the learning block were 3

342 no-shift sub-blocks of 4 trials each wherein the target location was not changed and 343 the original target stayed on the screen (no TPE). Before each of these sub-blocks, 344 subjects were so informed and were instructed to reach to the original target. At the 345 end of the sub-block, subjects were once again told that the target would start 346 "jumping" and they should reach to the new target as before (Figure 1C).

TPEs stimulated intentional changes in reach direction

We first examined the deviation in hand angle from the original target direction across the learning trials. These changes were quite idiosyncratic, with some subjects showing a rapid (within a few learning trials) shift of hand direction towards the new target while others continuing to aim towards the original target for a number of trials before abruptly switching their aim towards the new target (Figure 2A). Hardly any subject showed a gradual, progressive change in hand direction. The more steady trial-by-trial change in the group mean (Figure 2B, blue), therefore, resulted from averaging. Differences in subject performance during the initial learning phase were also evident as highly variable hand deviations (Figure 2C). Despite these early differences, all subjects learned to aim directly towards the new target location by the end of the learning block (Figure 2C, mean hand deviation during the late learning stage $\left.=44.65 \pm 1.13^{\circ}\right)$. Thus, subjects were able to account for the TPE and adjust their reach direction accordingly.

Performance on the no-shift sub-blocks allowed us to probe the mechanism through which subjects learned to cancel the TPE. On these trials, subjects aimed directly towards the original target as instructed, and hand deviation fell to near zero on each of the sub-blocks (Figure 2D, first: $0.947 \pm 0.633^{\circ}, 99 \% \mathrm{Cl}=[-0.797,2.691]$, second: $0.945 \pm 0.576^{\circ}, 99 \% \mathrm{Cl}=[-0.643,2.533]$, third: $\left.1.711 \pm 1^{\circ}, 99 \% \mathrm{Cl}=[-1.044,4.466]\right)$ Post-learning after-effects were also absent with near zero hand deviation $\left(\right.$ mean \pm SE $\left.=1.022 \pm 0.497^{\circ}, 99 \% \mathrm{Cl}=[-0.349,2.393]\right)$ on early washout trials Figure 2D). Statistically, there was no difference between the late baseline trials, no-shift sub-blocks and early washout trials $(F(2.656,77.022)=1.219, p=0.307)$. This immediate unlearning indicated that the change in hand angle on the target-shift 
373 trials of the learning block was due to the use of an intentional strategy that could be

374 "turned off" upon instruction.

375

376

377

378

379

380

381

382

383

384

385

386

387

388

389

390

391

392

393

394

395

396

397

398

399

400

401

402

403

404

405

We next predicted that if subjects were using a deliberative strategy to aim towards the displaced target on the learning trials, their reaction times (RT) would be higher on those trials. We observed (Figures 2E, 2F) that while baseline RT was close to $400 \mathrm{~ms}$ (mean $\pm S E=397 \pm 11 \mathrm{~ms}$ ), it increased to about $550 \mathrm{~ms}$ on the target-shift trials (mean $\pm S E=556 \pm 21 \mathrm{~ms}$ ), a change that was clearly statistically significant (Wilcoxon signed-rank test, $\mathrm{W}=0, \mathrm{p}<0.001$, matched ranked biserial correlation = 1.000; estimation statistics: $95 \% \mathrm{Cl}$ of paired mean difference $=[0.127,0.203], \mathrm{p}<$ 0.001 for two-sided permutation t-test with 5000 bootstrap samples). Critically, on the no-shift sub-blocks, when subjects were informed that the target would not jump, their RT dropped considerably compared to the immediately prior learning trials (Figures 2E, 2G). Likewise, RT on the early washout trials also smaller than the late learning trials. There was no difference in the magnitude of $\mathrm{RT}$ reduction across the 3 no-shift sub-blocks and the early washout trials (Figure $2 \mathrm{G}, \mathrm{F}(3,87)=0.1314, \mathrm{p}=$ $\left.0.941, \omega^{2}=0\right)$. This pattern - an increase in RT when the target location shifted, but an immediate reduction when it did not - bolstered the view that the TPE-mediated learning on the target-shift trials was deliberate in nature.

\section{Savings occurred upon re-exposure to TPEs}

We next probed for savings, and posited that if savings reflects the recall of learned strategies, it should occur when subjects are re-exposed to the TPEs. We found that hand angle changes from the original to the new target direction occurred over far fewer trials than initial learning, suggesting savings from prior learning (Figure 2B, pink). Hand deviation was much larger during the early phase of the savings block than the learning block (Figure 3A, Wilcoxon signed rank test, $W=9, p<0.001$, matched ranked biserial correlation $=-0.961)$. Additionally, on the no-shift subblocks, subjects again demonstrated rapid disengagement of learning. Hand deviation was now close to zero again (Figure 3B), and there were no significant differences relative to the late washout trials $\left(F(3,87)=1.167, p=0.327, \omega^{2}=\right.$ 0.003). As was the case during learning, RT increased on the target-shift trials of the savings block, but also dropped to late washout levels on the no-shift sub-blocks 
406 (Figure 3C). Collectively, the results of this first experiment indicated that in the 407 absence of SPEs, TPEs are compensated via intentional mechanisms that are 408 responsive to verbal instruction. The use of such strategies also promotes savings, 409 suggesting that exposure to SPEs may not be necessary for this purpose.

\section{TPE-mediated changes in movement direction were verbalizable}

412 In our second experiment (Figure 1D), we sought to directly analyze how subjects

413 explicitly formulate their reaching strategy while adapting to TPEs. Unlike Experiment

414 1, which used an indirect, exclusion method, in Experiment 2 we asked subjects to 415 directly report their aiming angle on each trial with the help of a ring of equiangular 416 numerical landmarks concentric to the start position (Taylor et al., 2014). Subjects 417 performed reaches to targets that "jumped" $45^{\circ}$ counterclockwise on learning trials; 418 they were also informed about the occurrence of the jumps and instructed to reach to 419 the new target location. On washout trials, they were again informed that the targets 420 would not jump and they should reach to the original target.

422 Subjects started the learning block typically by reporting landmark number "0", which 423 corresponded to the original target. All subjects eventually began reporting, and 424 persisted with, their reports of the angle corresponding to the new location of the 425 target, i.e., landmark number "9" (Figure 4A, yellow). These verbal reports appeared 426 to show higher variance during the early phase of learning, and low variance towards 427 the end, consistent prior observations (Taylor et al., 2014). We further quantified this 428 behavior by calculating the probability of aim change across trials of the learning 429 block (Figure 4B). This probability was much greater during the early phase of 430 learning (reaching a peak value of $\sim 70 \%$ on the sixth learning trial), and dropped to 431 approximately 0 by the end of the learning block. This was also statistically 432 confirmed as a significant difference in the aim change probability values of the early 433 and late learning phases (Wilcoxon signed rank test, $W=40.5, p=0.025$, matched 434 ranked biserial correlation $=0.8$ )

436 Critically, the actual hand angle closely mirrored the reported aim. Subjects started 437 aiming their hand (Figure 4A, blue) towards the new target early on and attained 438 complete compensation by the end of the learning block (mean $\pm S E=46.194 \pm 0$. 
$\left.913^{\circ}\right)$; this change was statistically robust $(t(9)=-12.116, p<0.001$, Cohen's $d=-$

440 3.831). More importantly however, there was no significant difference between the

441 reported aiming angle and the actual hand angle at the beginning $(\mathrm{t}(9)=0.723, \mathrm{p}=$

4420.488 , Cohen's $d=0.229)$ or at the end $(t(9)=1.541, p=0.158$, Cohen's $d=0.487$ )

443 of the learning block, indicating that subjects actually aimed in the direction that they

444 reported they would.

The difference between the reported aim and the actual hand angle provides a marker for implicit learning. We computed average implicit learning (Figure 4A, green), and found that it was near zero during the early (mean $\pm S E=2.744 \pm 3.793^{\circ}$, $99 \% \mathrm{Cl}=[-9.581,15.069])$ as well as late $\left(\right.$ mean $\pm \mathrm{SE}=1.319 \pm 0.856^{\circ}, 99 \% \mathrm{Cl}=[-$ $1.463,4.101])$ phases of the learning block. This indicated that subjects did not learn implicitly at all, and were using explicit strategies to compensate for the error that the

452 target-shift induced. To confirm this, we also examined after-effects in the washout 453 block (Figure 4A). We again found that subjects were able to immediately "unlearn" 454 when informed that the target position would not change. Subjects not only reported 455 landmark number "0" (corresponding to the original target location) right away, but 456 their hand deviation on early washout trials also dropped to near zero $2.023 \pm 0.858^{\circ}$ $457(99 \% \mathrm{Cl}=[-0.765,4.811])$. All in all, these results advocated that subjects primarily 458 relied on the use of consciously accessible, volitional strategies to compensate for 459 the target-shift-induced TPE.

Finally, we observed clear savings when subjects were re-exposed to the target jumps following washout. Subjects reported the new target location and also moved their hand towards it earlier (Figure 4C, pink) than in the training block (Figure 4C, blue). The variability in hand angle in the savings block was also low, suggesting that all subjects were able to successfully employ the previous strategy quite quickly. The change in the reported $(t(9)=-12.142, p<0.001$, Cohen's $d=-3.84)$ as well as actual hand angles $(t(9)=-13.223, p<0.001$, Cohen's $d=-4.182)$ during the early phase of the savings block were much larger compared to initial learning, indicating clearly that savings was present (Figure 4D). This result once again indicated that savings does not require experience of an SPE, and is likely driven by the recall of previously employed re-aiming processes. 
472

473 Changes in reach direction were sensitive to TPE magnitude.

474 Recent work suggests that while implicit learning is relatively rigid and insensitive to

475 perturbation size, strategy use engenders greater flexibility (Bond and Taylor, 2015).

476 We therefore hypothesized that the change in hand angle would scale with the size

477 of the TPE rather than simply have a binary effect. We tested this idea in our third

478 experiment by adopting a design similar to experiment 1 (Figure $1 \mathrm{C}$ ) but assigning

479 subjects to 3 groups that differed based on TPE size $\left(15^{\circ}, 30^{\circ}\right.$, or $\left.60^{\circ}\right)$. Task

480 instructions and their schedule remained identical to experiment 1 . All three groups

481 changed their reach direction to account for the shift in target location. While hand

482 deviation during early learning was not different between the groups $(F(2,32)=$

$4832.609, p=0.09, \omega^{2}=0.084$ ), it was clearly so at the end of learning ( $15^{\circ}$ group:

$48412.032 \pm 2.076^{\circ}, 30^{\circ}$ group: $=29.458 \pm 1.426^{\circ}, 60^{\circ}$ group: $54.239 \pm 2.261^{\circ}, F(2,32)=$

$485117.274, p<0.001, \omega^{2}=0.869$, compare asymptote phase of Figures 5A, 5B and

$4865 \mathrm{C})$. This scaling indicated that the adaptive response was indeed sensitive to the

487 size of the TPE.

488

\section{Strategies for compensating small versus large TPEs were dissociable.}

490

Interestingly, we observed that for the $15^{\circ}$ group, the average compensation was

491

less complete than the other groups. By the end of learning, this group had

492

compensated only $\sim 80 \%$ of the TPE (mean \pm SE $=80.21 \pm 13.84 \%$ ), while the $30^{\circ}$ and

493

$60^{\circ}$ groups had compensated more than $90 \%$ (mean \pm SE $=98.19 \pm 4.75 \%$ and

494

$90.4 \pm 3.77 \%$ for the $30^{\circ}$ and $60^{\circ}$ groups respectively). Importantly, this was not

because subjects in the $15^{\circ}$ group had achieved a "good enough" solution, i.e., they

496 were able to hit the shifted target without having to fully compensate for the TPE.

497 Considering that the target diameter was $1.5 \mathrm{~cm}$, the cursor would hit the target if the 498 hand angle changed by $12.11^{\circ}$ for a $15^{\circ}$ shift. However, we found that even at the 499 end of learning, subjects did not reach this threshold on more than $50 \%$ of the trials 500 (mean $=52.27 \%$ ). This indicated that compensation indeed remained incomplete in 501 this group. We additionally observed that the average variance in (normalized) hand 502 direction during the learning block was greater following the $15^{\circ}$ TPE (Figure 5D).

503 These patterns in the data motivated a finer analysis, wherein we probed whether 
504 the manner in which subjects responded to the small TPE $\left(15^{\circ}\right)$ differed from the

505 larger ones $\left(30^{\circ}\right.$ and $\left.60^{\circ}\right)$.

506

507 We first focused on the RT data. While RT increased on the learning trials for all 508 groups relative to baseline, this increase was not uniform (Figure 5E). We observed

509 a dichotomous response: a small increase for the $15^{\circ}$ group (mean $\pm \mathrm{SE} \Delta \mathrm{RT}=63 \pm 21$

$510 \mathrm{~ms})$, but larger increases for the $30^{\circ}(172 \pm 38 \mathrm{~ms})$ and $60^{\circ}(163 \pm 14 \mathrm{~ms})$ groups. This

511 was statistically confirmed via a significant group difference in a Welch's ANOVA

$512\left(F(2,19.144)=7.702, p=0.004, \omega^{2}=0.18\right)$. Post-hoc tests revealed not only that

513 the RT increase was much more for the $30^{\circ}(p=0.022)$ and $60^{\circ}(p=0.037)$ groups

514 relative to the $15^{\circ}$ group, but also that these two larger TPE groups did not differ

515 from each other $(p=0.97)$. RT differences between the $15^{\circ}$ and $60^{\circ}$ groups were

516 confirmed using estimation statistics $(95 \% \mathrm{Cl}$ of unpaired mean difference $=[0.024$,

5170.188 ], $p=0.026$ for two-sided permutation t-test with 5000 bootstrap samples), as

518 were the differences between the $30^{\circ}$ and $60^{\circ}$ groups $(95 \% \mathrm{Cl}$ of unpaired mean

519 difference $=[0.048,0.144], p=0.001$ for two-sided permutation t-test with 5000

520 bootstrap samples). Likewise, a Bayesian independent samples t-test, which yielded

521 a $\mathrm{BF}_{10}$ value of 0.38 , provided support to the hypothesis that RTs of the $30^{\circ}$ and $60^{\circ}$

522 groups were not different from each other; the same was confirmed using estimation

523 methods $(95 \% \mathrm{Cl}$ of unpaired mean difference $=[-0.1,0.061], p=0.663$ for two-sided

524 permutation t-test with 5000 bootstrap samples). In sum, these patterns indicated

525 that RT did not scale uniformly with error size.

526

527 Another hint supporting a potential dissociation in strategies for compensating small

528 versus large TPEs came from the hand angle data of the no-shift sub-blocks,

529 although, admittedly, this was less clear than the variability, amount of learning and

$530 \mathrm{RT}$ results reported above. Consider the behavior of the $15^{\circ}$ group first. For these

531 subjects, we observed that the mean hand deviation on the first no-shift sub-block

532 was close to zero $\left(-0.222 \pm 0.845^{\circ}, 99 \% \mathrm{Cl}=[-2.901,2.456]\right)$. However, hand

533 deviation on the subsequent no-shift sub-blocks did not return to these levels (Figure

$5345 \mathrm{~F})$. Specifically, hand deviation on the third no-shift sub-block was larger than that

535 on the first such sub-block $(t(10)=-2.6651, p=0.0237$, Cohen's $d=-0.8036)$.

536 Furthermore, the deviation on the early washout trials remained (marginally) 
elevated relative to the first no-shift sub-block (paired t-test, $\mathrm{t}(10)=-2.2265, \mathrm{p}=$

5380.0501 , Cohen's $d=-0.6713)$, but was not different from that on the last such sub-

539 block (paired t-test, $t(10)=1.3732, p=0.1997$, Cohen's $d=0.414$ ). This suggested

540 that there was some tendency for the learned behavior to persist even after the

541 perturbation had been removed. Notably, this was also the case when we used

542 baseline uncorrected data for our analyses, suggesting that this result was not an

543 artifact of baseline bias elimination. It is however possible that some of these results

544 were influenced by a potential outlier who showed a hand deviation of approximately

$545-7^{\circ}$ on the first no-shift sub-block. When this subject was excluded, the the difference

546 in hand deviation on the first and last no-shift sub-block was borderline significant

547 with a medium-large effect size $(t(9)=-2.262, p=0.05$, Cohen's $d=-0.7154)$. In the

548 Bayesian realm, the same comparison (without the outlier) yielded a $\mathrm{BF}_{10}$ value of

5491.7627 (error $=0.0018 \%$ ), which provided anecdotal evidence in favor of the

550 hypothesis that hand deviation on the last no-shift sub-block was greater than that on

551 the first such sub-block in this group. This difference may therefore be interpreted

552 with some caution.

553

554 In contrast, there was clearly no difference in hand deviation between the first and

555 last no-shift sub-blocks for the $30^{\circ}$ (Figure 5G, $\mathrm{t}(11)=-1.8882, \mathrm{p}=0.0856$, Cohen's

$556 \mathrm{~d}=-0.5451$ ) or $60^{\circ}$ (Figure $5 \mathrm{H}, \mathrm{t}(11)=0.1659, \mathrm{p}=0.8713$, Cohen's $\mathrm{d}=0.0479$ )

557 groups. Likewise, we found no difference between the early washout trials and the

558 first no-shift sub-block for the $30^{\circ}$ group ( $t(11)=-1.3371, p=0.2082$, Cohen's $d=$ -

559 0.386). This was also the case for the $60^{\circ}$ group $(\mathrm{t}(11)=1.2449, \mathrm{p}=0.239$, Cohen's

$560 d=0.3594)$. This suggested that these subjects immediately and consistently

561 returned to earlier performance levels across all no-shift sub-blocks as well as the

562 washout block. Collectively, the distinct trends in variability, fraction of TPE

563 compensated, and RT and hand deviation data suggested that smaller TPEs $\left(15^{\circ}\right.$ in

564 our case) might be compensated differently relative to larger ones $\left(30^{\circ}, 45^{\circ}\right.$

565 (experiment 1) and 60\%).

566

567 Finally, we observed that when re-exposed to target-shifts after washout, subjects in

568 all groups exhibited savings, as was the case in experiments 1 and 2. Subjects

569 compensated for the imposed TPE by directing their hand towards the new target

570 faster than they did in the training block. This expression of savings was also reliably 
571 captured via our statistical comparisons: mean hand angle was clearly larger on the

572 early savings trials compared to the early learning trials for each group (Figure 5I,

$57315^{\circ}$ group: $\mathrm{t}(10)=-5.226, \mathrm{p}<0.001$, Cohen's $\mathrm{d}=-1.576 ; 30^{\circ}$ group: $\mathrm{t}(11)=-6.952$,

$574 p<0.001$, Cohen's $d=-2.007 ; 60^{\circ}$ group: $t(11)=-7.545, p<0.001$, Cohen's $d=-$

575 2.178).

576

Taken together, our results indicate that: 1) in the absence of an SPE, adaptive responses to consistently presented TPEs occur in the form of volitional strategies, 2) these strategies could be sensitive to the size of the TPE, and 3) strategy use facilitates savings; a history of exposure to SPEs is not needed for savings to occur.

\section{DISCUSSION}

583

In a series of experiments, we probed how the motor system responds to recurring TPEs. We demonstrate that TPEs are compensated entirely via intentional, explicitlyaccessible strategies, reflecting enhanced action selection. A fundamental question is whether such compensation constitutes "adaptive" behavior at all. Insofar as adaptation is defined as a change in motor behavior following exposure to a perturbing environment, the answer is yes. However, if it is viewed more narrowly as a performance change set in motion specifically by SPEs, then perhaps no. We imposed no SPE, and the change in motor output was potentiated by a TPE elicited via a target shift.

594 There are many reasons to believe that this change was explicitly driven. In

595 Experiment 1, individual-level changes in hand direction were quite idiosyncratic and the group-level exponential trend emerged only as an artifact of averaging. This is not observed with implicit learning, wherein individual subjects also typically demonstrate exponential changes. Further, there was a substantial RT increase on target-shift trials, suggesting the engagement of time-consuming and deliberative mental processes (Fernandez-Ruiz et al., 2011; Haith et al., 2015; McDougle and

601 Taylor, 2019). Subjects also disengaged from the "learned" behavior immediately

602 upon instruction, with a concomitant drop in RT; such flexibility is a hallmark of 603 explicit but not implicit learning (Bond and Taylor, 2015). Relatedly, no after-effects 604 were evident on washout trials. In Experiment 2, subjects were able to precisely 
605

606

607

608

609

610

611

612

613

614

615

616

report the aiming location and also reach there, without any implicit change in their reach direction. Finally, Experiment 3 revealed that the asymptotic level of hand deviation was sensitive to TPE magnitude, unlike what has been observed with implicit learning (Kasuga et al., 2013; Morehead et al., 2017; Wei and Körding, 2009). Collectively, these observations reject the possibility that TPEs, at least as imposed through shifts in target location, are compensated implicitly. Rather, our results strongly indicate that they set in motion explicitly accessible, intentional aiming strategies.

\section{Experiment 3 suggested the intriguing possibility that strategies employed to} compensate small versus large TPEs could be distinct. Large target-shifts could be compensated in two ways. First, subjects could mentally rotate reach plans for moving to the initially presented target (Fernandez-Ruiz et al., 2011; McDougle and Taylor, 2019), underpinned by premotor and M1 circuits (Georgopoulos et al., 1989; Kosslyn et al., 1998). A key prediction of this hypothesis however is that RT should scale with perturbation magnitude, which did not bear out in our data. Additionally, mental rotation can lead to incomplete learning (McDougle and Taylor, 2019) whereas we observed more complete compensation for larger errors.

A compelling alternative then is that subjects learn to re-aim by actually learning the task structure and using it to deliberatively evaluate potential actions by mentally simulating their consequences. Specifically, actions are guided by representations of outcomes they produce given the state of the environment and what these outcomes are worth, as in model-based reinforcement learning (Daw et al., 2005; Dickinson and Balleine, 1994; Doll et al., 2012; Doya, 2000). It is known that despite being time-consuming, such goal-directed algorithms are highly flexible and can be adjusted to account for changes induced via outcome revaluation, and environment and goal changes. The longer RTs on the shift trials and the rapid, instruction-driven disengagement of the strategy on the no-shift trials, are highly in line with this notion.

In contrast to model-based control, small TPEs likely set in motion different mechanisms. When the target-shifts were small, we observed greater variability during early learning, a small undershoot during the asymptotic phase, a smaller RT increase on shift trials, and persistence of the learned behavior during the late no- 
639

640

641

642

643

644

645

646

647

648

649

650

651

652

653

654

655

656

657

658

659

660

661

662

663

664

665

666

667

668

669

670 Why might strategies differ for learning from small versus large TPEs? One reason

671

672

shift trials (though this last result was not as clear-cut as the others). We suggest that this occurs because subjects might employ a "model-free" strategy (Kaelbling et al., 1996; Sutton and Barto, 1998) to counter small TPEs. That is, they explore the solution space for a movement that cancels the TPE and then repeat it as it leads to successful or rewarding outcomes. Such a strategy engenders higher variability initially, including a few trials on which subjects move away from the direction of the shift (Figure 2A, first few learning trials). Furthermore, repetition yields robust stimulus-response associations, leading to the execution of the successful action whenever a (small) target-shift occurs. Such responses are computationally frugal, but they are also inflexible, leading to a continued expression of the learned, "habitual" behavior (Graybiel, 2008), a hint of which was seen on the late no-shift and early washout trials in the $15^{\circ}$ shift group.

Could it rather be that adaptive responses to small TPEs ( $15^{\circ}$ in our case) are driven by some kind of implicit process, like for SPEs? We posit that this is not the case.

Diedrichsen et al. (2005) examined changes in motor output following exposure to a $12^{\circ}$ error elicited either via a target-shift (TPE) or a visuomotor rotation (SPE). They reported that unlike the SPE, the TPE-mediated change did not carry signatures of implicit learning. Additionally, recent work (Oza et al., 2020) has shown that when explicitly instructed to ignore a consistently occurring $10^{\circ}$ shift in target location, subjects are able to do so quite well. A similar sensitivity to instruction has been reported by for even smaller TPEs (Tsay et al., 2021). This would not be expected from a system undergoing implicit recalibration (Mazzoni and Krakauer, 2006; Morehead et al., 2017). Finally, it has been proposed that re-exposure to a perturbing environment produces an attenuation in the implicit response, and an enhancement of the strategic component that ultimately produces savings (Avraham et al., 2021). Savings was evident in our $15^{\circ}$ target-shift group as well; since we did not induce SPEs, this could be attributed only to a strategic process. As such, we suggest that when TPEs are small, subjects choose to aim to the new target location that gets cached or memorized with practice.

could be that model-free motor exploration can be very slow in terms of the number of attempts needed to arrive at the solution, even when the task structure is simple to 
673 learn. This strategy may therefore be functionally quite limited. When the limits of

674 exploration are reached (i.e., when TPE magnitude is beyond tolerable levels), the

675 sensorimotor system might abandon this strategy in favor of a new one that involves

676 extracting as much information about the environment as possible, and selecting

677 actions that account for changes in it. Notably, a dissociation for dealing with small

678 versus large TPEs has been shown in studies of the behavioral (Day and Lyon,

679 2000; Desmurget et al., 2004; Mutha et al., 2008) and neural (Day and Brown, 2001;

680 Desmurget et al., 2001) correlates of online, feedback-mediated motor corrections.

681 Our results suggest that a similar dichotomy could hold for feedforward processes as 682 well.

683

684 Our experiments also clearly brought forth savings when subjects were re-exposed

685 to the target-shift following washout. Since we never imposed an SPE, this result

686 indicates that a history of exposure to SPEs is likely not needed for a latent memory

687 that facilitates faster re-learning to be expressed. This nicely converges with recent

688 work (Leow et al., 2020) demonstrating savings even when subjects never adapt to

689 an SPE, but are exposed to a TPE before the SPE (and the solution to cancel both is

690 the same in hand space). Our experimental design allowed us to isolate the TPE,

691 and its disentanglement from the SPE enabled greater certainty about the

692 determinants of latent memories in sensorimotor learning. We suggest, in

693 conjunction with other results (Haith et al., 2015; Huberdeau et al., 2015; Morehead

694 et al., 2015), that SPE-specific implicit mechanisms are not a significant contributor

695 to savings.

696

697 How do strategic processes foster savings? First, stimulus-response associations

698 such as those formed for smaller target-shifts, could get directly cached in memory

699 and retrieved when appropriate. Such retrieval requires less time and little cognitive

700 effort (Logan, 1988). It is not clear whether model-based simulations of action

701 outcomes employed to counter larger target-shifts are also cached and later

702 retrieved without any additional planning. But, another way in which savings could

703 emerge from model-based control is that mental simulations could be used to train a

704 model-free process to reduce computational cost in the long run; the possibility for

705 such an interaction has been raised before (Daw et al., 2011). This is essentially a

706 practice-mediated transition from goal-directed to automatic, habitual behavior. Such 
707 a deliberate-to-automatic change likely explains why savings occurs even when

708 preparation time is constrained but subjects are overtrained (Huberdeau et al.,

709 2019).

710

711 Model-based and model-free mechanisms set in motion by large and small TPEs

712 respectively could be supported by distinct neural networks. Numerous rodent

713 studies have shown that model-free learning relies on dorsolateral striatum (posterior

714 putamen in primates). This region is richly irrigated by inputs from sensorimotor

715 cortex, and is essential for the formation and expression of stimulus-response

716 associations (Devan et al., 2011; Graybiel, 2008; Yin and Knowlton, 2006). In

717 contrast, goal-directed, model-based actions require intact processing in

718 dorsomedial striatum (caudate and rostral putamen in primates), which receives

719 abundant inputs from prefrontal cortical areas (Redgrave et al., 2010; Yin et al.,

720 2005). This dissociation is evident in humans as well, with greater activation in the

721 anterior caudate for model-based control (Tanaka et al., 2008), and caudal putamen

722 for stimulus-response mediated behavior (Tricomi et al., 2009). Importantly, it has

723 been shown that repeated practice leading to a shift from goal-directed to more

724 direct stimulus-response control, is also associated with a transition in activation in

725 rostromedial (associative) to caudolateral (sensorimotor) striatum (Jueptner et al.,

726 1997; Lehéricy et al., 2005). In our case, such a shift towards striatal circuits

727 supporting automaticity could occur when large TPEs are repeatedly countered. This

728 activity could support long-term motor memories that eventually give rise to savings.

729 Strengthening this view is the finding that Parkinson's disease patients, who show

730 impaired stimulus-response learning (Frank et al., 2004; Rutledge et al., 2009;

731 Shohamy et al., 2006), also show deficient savings (Bédard and Sanes, 2011; Leow

732 et al., 2013). When a TPE is accompanied by a limb-related SPE, a parallel network

733 involving the cerebellum and parietal cortex is likely activated to recalibrate an

734 internal model of the physics of the limb. How these two neural systems cooperate

735 (or compete) to forge overall adaptive behavior should be an exciting area for future

736 investigation. 
Figure 1. Experimental setup and tasks (A) Subjects performed reaching movements on a digitizing tablet using a handheld stylus while looking into a mirror placed between the tablet and a horizontally mounted display. Start positions, targets, and a feedback cursor displayed on the screen were reflected in the mirror. (B) Target locations and sample hand trajectories on early (solid) and late (dotted) learning trials. The original target has been blurred, while the new, shifted target is shown in solid colors. (C) Task protocol for experiments 1 and 3. The baseline block was followed by learning trials on which the target-shift created a TPE. This was followed by washout and a final "savings" block on which subjects re-experienced the target-shifts. In Experiment 1, the target-shift was $45^{\circ}$ (solid line), while in Experiment 3 , it was $15^{\circ}, 30^{\circ}$ or $60^{\circ}$ (dotted lines) for different groups. In both experiments, 3 "no-shift" sub-blocks of 4 trials each were embedded during learning and savings trials; their location is shown using black bars. Verbal instructions were given every time the target conditions were about to change. (D) In Experiment 2, subjects again performed 4 blocks of trials, but without the no-shift sub-blocks. Additionally, the original target was presented with a ring of numbers as shown on the right. Before each trial, subjects reported the approximate number they would reach to. The original target location always corresponded to number " 0 ", while the shifted target corresponded to " 9 ". The ring appeared with the original target and disappeared with the presentation of the new target.

Figure 2. TPEs are compensated through intentional strategies (A) Hand deviation (relative to the original target) on the late baseline and first 28 learning trials (each subject shown using a different color). The profile of two subjects is bolded to highlight the variability across subjects. One of them changed movement direction quite early during learning while the other did so quite late. (B) Groupaveraged hand deviation across trials. Shaded regions denote SEM. Learning (blue) and savings (pink) data are superimposed for ease of comparison; trial 1 corresponds to the first learning trial (or the first savings trial). No-shift trials are highlighted using grey bands. Hand deviation on late baseline, no-shift and early washout trials is shown using open circles. (C) Mean hand deviation during early and late learning. Dots represent individual subjects. Error bars are SEM. (D) Mean hand 
771 deviation on the no-shift sub-blocks and early washout trials. Dots are individual

772 subjects. Error bars are SEM. (E) Group-averaged RT across trials. Shaded regions

773 denote SEM. No-shift sub-blocks are highlighted in grey. RT on no-shift trials as well

774 as late baseline and early washout trials, is shown in open circles. (F) Mean RT in

775 the baseline and learning blocks. Dots represent individual subjects. Error bars are

776 SEM (G) Change in RT on the no-shift and early washout trials relative to the

777 immediately prior learning trial. Dots represent individual subjects. Error bars are

778 SEM.

779

780

781

Figure 3. Strategy-use results in savings. (A) Mean hand angle during the early

782 learning (blue) and early savings (pink) phase. Dots represent individual subjects. Error bars are SEM. (B) Mean hand angle on late washout and no-shift trials of the savings block. Dots represent individual subjects. Error bars are SEM. (C) Average RT on late washout and no-shift trials of the savings block. Dots are individual subjects. Error bars are SEM.

Figure 4. Directional changes in response to TPEs are verbalizable. (A) Groupaveraged hand deviation (blue), reported aiming direction (yellow), and the implicit component (green) across trials. Shaded regions denote SEM. (B) Mean trial-wise probability of aim change across learning trials. Shaded regions are SEM. (C) Group-averaged hand deviation across trials. Shaded regions denote SEM. Learning (blue, same as in A) and savings (pink) data are superimposed for ease of comparison; trial 1 corresponds to the first learning trial (or first savings trial). Late baseline and early washout trials are shown using open circles. (D) Mean hand deviation on early learning and early savings trials. Dots represent individual subjects. Error bars are SEM.

Figure 5. Strategies employed to compensate small versus large TPEs are likely dissociable. Group-averaged baseline-corrected hand deviation across trials for the (A) $15^{\circ}$, (B) $30^{\circ}$, and (C) $60^{\circ}$ target-shift groups. Shaded regions denote

801 SEM. Remaining details are same as Figure 2A. (D) Mean variance in normalized

802 hand deviation for the 3 groups. No error bars are shown since this measure was

803 calculated for the entire group, not individual subjects. (E) Mean RT on baseline and 804 learning trials. Dots are individual subjects. Error bars are SEM. (F, G, H) Mean 
805 baseline-corrected hand angle on the no-shift sub-blocks embedded within the 806 learning block, early washout trials, and no-shift sub-blocks of the savings block for 807 the (F) $15^{\circ},(\mathbf{G}) 30^{\circ}$, and (H) $60^{\circ}$ target-shift groups. (I) Mean hand deviation on the 808 early learning and early savings trials for the three groups. Dots represent individual 809 subjects. Error bars are SEM. 


\section{REFERENCES}

Anguera JA, Reuter-Lorenz PA, Willingham DT, Seidler RD (2010) Contributions of spatial working memory to visuomotor learning. J Cogn Neurosci 22:1917-1930.

Avraham G, Morehead JR, Kim HE, Ivry RB (2021) Reexposure to a sensorimotor perturbation produces opposite effects on explicit and implicit learning processes. PLoS Biol 19.

Bédard P, Sanes JN (2011) Basal ganglia-dependent processes in recalling learned visual-motor adaptations. Exp Brain Res 209:385-393.

Bond KM, Taylor JA (2015) Flexible explicit but rigid implicit learning in a visuomotor adaptation task. J Neurophysiol 113:3836-3849.

Bostan AC, Strick PL (2018) The basal ganglia and the cerebellum: nodes in an integrated network. Nat Rev Neurosci 19:338-350.

Clower DM, Hoffman JM, Votaw JR, Faber TL, Woods RP, Alexander GE (1996) Role of posterior parietal cortex in the recalibration of visually guided reaching. Nat 19963836601 383:618-621.

Coltman SK, Cashaback JGA, Gribble PL (2019) Both fast and slow learning processes contribute to savings following sensorimotor adaptation. J Neurophysiol 121:1575-1583.

Daw ND, Gershman SJ, Seymour B, Dayan P, Dolan RJ (2011) Model-based influences on humans' choices and striatalprediction errors. Neuron 69:1204.

Daw ND, Niv Y, Dayan P (2005) Uncertainty-based competition between prefrontal and dorsolateral striatal systems for behavioral control. Nat Neurosci 8:17041711.

Day BL, Brown P (2001) Evidence for subcortical involvement in the visual control of human reaching. Brain 124:1832-1840.

Day BL, Lyon IN (2000) Voluntary modification of automatic arm movements evoked by motion of a visual target. Exp Brain Res 130:159-168.

Della-Maggiore V, Malfait N, Ostry DJ, Paus T (2004) Stimulation of the posterior parietal cortex interferes with arm trajectory adjustments during the learning of new dynamics. J Neurosci 24:9971-9976.

Desmurget M, Grafton ST, Vindras P, Gréa H, Turner RS (2004) The basal ganglia network mediates the planning of movement amplitude. Eur J Neurosci 19:2871-2880.

Desmurget M, Gréa H, Grethe JS, Prablanc C, Alexander GE, Grafton ST (2001) Functional anatomy of nonvisual feedback loops during reaching: a positron emission tomography study. J Neurosci 21:2919-28.

Devan B, Hong N, McDonald R (2011) Parallel associative processing in the dorsal striatum: segregation of stimulus-response and cognitive control subregions. Neurobiol Learn Mem 96:95-120.

Dickinson A, Balleine B (1994) Motivational control of goal-directed action. Anim Learn Behav 1994221 22:1-18.

Diedrichsen J, Hashambhoy Y, Rane T, Shadmehr R (2005) Neural correlates of reach errors. J Neurosci 25:9919-9931.

Doll BB, Simon DA, Daw ND (2012) The ubiquity of model-based reinforcement learning. Curr Opin Neurobiol 22:1075-1081.

Doya K (2000) Complementary roles of basal ganglia and cerebellum in learning and motor control. Curr Opin Neurobiol 10:732-739.

Fernandez-Ruiz J, Wong W, Armstrong IT, Flanagan JR (2011) Relation between reaction time and reach errors during visuomotor adaptation. Behav Brain Res 219:8-14. 
860

861

862

863

864

865

866

867

868

869

870

871

872

873

874

875

876

877

878

879

880

881

882

883

884

885

886

887

888

889

890

891

892

893

894

895

896

897

898

899

900

901

902

903

904

905

906

907

908

909

Flament D, Ellermann JM, Kim SG, Ugurbil K, Ebner TJ (1996) Functional magnetic resonance imaging of cerebellar activation during the learning of a visuomotor dissociation task. Hum Brain Mapp 4:210-226.

Frank M, Seeberger L, O'reilly R (2004) By carrot or by stick: cognitive reinforcement learning in parkinsonism. Science 306:1940-1943.

Georgopoulos AP, Lurito JT, Petrides M, Schwartz AB, Massey JT (1989) Mental rotation of the neuronal population vector. Science (80- ) 243:234-236.

Graybiel AM (2008) Habits, rituals, and the evaluative brain. Annu Rev Neurosci 31:359-387.

Haith AM, Huberdeau DM, Krakauer JW (2015) The influence of movement preparation time on the expression of visuomotor learning and savings. $\mathrm{J}$ Neurosci 35:5109-5117.

Herzfeld DJ, Vaswani PA, Marko MK, Shadmehr R (2014) A memory of errors in sensorimotor learning. Science 345:1349-1353.

Huang VS, Haith A, Mazzoni P, Krakauer JW (2011) Rethinking motor learning and savings in adaptation paradigms: model-free memory for successful actions combines with internal models. Neuron 70:787-801.

Huberdeau DM, Haith AM, Krakauer JW (2015) Formation of a long-term memory for visuomotor adaptation following only a few trials of practice. J Neurophysiol 114:969-977.

Huberdeau DM, Krakauer JW, Haith AM (2019) Practice induces a qualitative change in the memory representation for visuomotor learning. J Neurophysiol 122:1050-1059.

Jueptner M, Stephan KM, Frith CD, Brooks DJ, Frackowiak RSJ, Passingham RE (1997) Anatomy of Motor Learning. I. Frontal Cortex and Attention to Action. https://doi.org/101152/jn19977731313 77:1313-1324.

Kaelbling LP, Littman ML, Moore AW (1996) Reinforcement learning: A survey. J Artif Intell Res 4:237-285.

Kasuga S, Hirashima M, Nozaki D (2013) Simultaneous Processing of Information on Multiple Errors in Visuomotor Learning. PLoS One 8:e72741.

Kim HE, Parvin DE, Ivry RB (2019) The influence of task outcome on implicit motor learning. Elife 8.

Kosslyn SM, DiGirolamo GJ, Thompson WL, Alpert NM (1998) Mental rotation of objects versus hands: Neural mechanisms revealed by positron emission tomography. Psychophysiology 35:151-161.

Kumar A, Panthi G, Divakar R, Mutha PK (2020) Mechanistic determinants of effector-independent motor memory encoding. Proc Natl Acad Sci 202001179.

Lefumat HZ, Vercher J-L, Miall RC, Cole J, Buloup F, Bringoux L, Bourdin C, Sarlegna FR (2015) To transfer or not to transfer? Kinematics and laterality quotient predict interlimb transfer of motor learning. J Neurophysiol 114:27642774.

Lehéricy S, Benali H, Van de Moortele P, Pélégrini-Issac M, Waechter T, Ugurbil K, Doyon $J$ (2005) Distinct basal ganglia territories are engaged in early and advanced motor sequence learning. Proc Natl Acad Sci U S A 102:1256612571.

Leow L-A, de Rugy A, Loftus AM, Hammond G (2013) Different mechanisms contributing to savings and anterograde interference are impaired in Parkinson's disease. Front Hum Neurosci 7:55.

Leow LA, Marinovic W, de Rugy A, Carroll TJ (2020) Task errors drive memories that improve sensorimotor adaptation. J Neurosci 40:3075-3088. 
910

911

912

913

914

915

916

917

918

919

920

921

922

923

924

925

926

927

928

929

930

931

932

933

934

935

936

937

938

939

940

941

942

943

944

945

946

947

948

949

950

951

952

953

954

955

956

957

958

959
Leow LA, Marinovic W, de Rugy A, Carroll TJ (2018) Task errors contribute to implicit aftereffects in sensorimotor adaptation. Eur J Neurosci 48:3397-3409.

Logan GD (1988) Toward an Instance Theory of Automatization. Psychol Rev 95:492-527.

Martin TA, Keating JG, Goodkin HP, Bastian AJ, Thach WT (1996) Throwing while looking through prisms. I. Focal olivocerebellar lesions impair adaptation. Brain 119 ( Pt 4:1183-1198.

Mazzoni P, Krakauer JW (2006) An implicit plan overrides an explicit strategy during visuomotor adaptation. J Neurosci 26:3642-3645.

McDougle SD, Taylor JA (2019) Dissociable cognitive strategies for sensorimotor learning. Nat Commun 10:40.

Morehead JR, Qasim SE, Crossley MJ, Ivry R (2015) Savings upon re-aiming in visuomotor adaptation. J Neurosci 35:14386-14396.

Morehead JR, Taylor JA, Parvin DE, Ivry RB (2017) Characteristics of Implicit Sensorimotor Adaptation Revealed by Task-irrelevant Clamped Feedback. J Cogn Neurosci 29:1061-1074.

Mutha PK, Boulinguez P, Sainburg RL (2008) Visual modulation of proprioceptive reflexes during movement. Brain Res 1246:54-69.

Mutha PK, Sainburg RL, Haaland KY (2011) Critical neural substrates for correcting unexpected trajectory errors and learning from them. Brain 134:3647-61.

Oza A, Kumar A, Mutha PK (2020) Task Errors Do Not Induce Implicit Sensorimotor Learning. bioRxiv 2020.11.13.381285.

Popa LS, Streng ML, Ebner TJ (2017) Long-term predictive and feedback encoding of motor signals in the simple spike discharge of purkinje cells. eNeuro 4.

Redgrave $\mathrm{P}$, Rodriguez M, Smith Y, Rodriguez-Oroz MC, Lehericy S, Bergman $\mathrm{H}$, Agid Y, Delong MR, Obeso JA (2010) Goal-directed and habitual control in the basal ganglia: Implications for Parkinson's disease. Nat Rev Neurosci 11:760772.

Rutledge R, Lazzaro S, Lau B, Myers C, Gluck M, Glimcher P (2009) Dopaminergic drugs modulate learning rates and perseveration in Parkinson's patients in a dynamic foraging task. J Neurosci 29:15104-15114.

Sainburg RL, Ghez C, Kalakanis D (1999) Intersegmental dynamics are controlled by sequential anticipatory, error correction, and postural mechanisms. J Neurophysiol 81:1045-1056.

Scheidt R a, Conditt M a, Secco EL, Mussa-Ivaldi F a (2005) Interaction of visual and proprioceptive feedback during adaptation of human reaching movements. $J$ Neurophysiol 93:3200-3213.

Shadmehr R, Mussa-Ivaldi F a. (1994) Adaptive Task of Dynamics during Learning of a Motor. J Neurosci 74:3208-3224.

Shohamy D, Myers C, Geghman K, Sage J, Gluck M (2006) L-dopa impairs learning, but spares generalization, in Parkinson's disease. Neuropsychologia 44:774784.

Sutton RS, Barto AG (1998) Reinforcement Learning: An Introduction. Cambridge, MA: The MIT Press.

Tanaka SC, Balleine BW, O'Doherty JP (2008) Calculating Consequences: Brain Systems That Encode the Causal Effects of Actions. J Neurosci 28:6750.

Taylor J a, Ivry RB (2012) The role of strategies in motor learning. Ann N Y Acad Sci 1251:1-12.

Taylor JA, Krakauer JW, Ivry RB (2014) Explicit and implicit contributions to learning in a sensorimotor adaptation task. J Neurosci 34:3023-3032. 
960

961

962

963

964

965

966

967

968

969

970

971

972

973

974

975

976

977

978

979

980

981

982

983
Tricomi E, Balleine BW, O'Doherty JP (2009) A specific role for posterior dorsolateral striatum in human habit learning. Eur J Neurosci 29:2225-2232.

Tsay JS, Haith AM, Ivry RB, Kim HE (2021) Distinct Processing of Sensory Prediction Error and Task Error during Motor Learning. bioRxiv 2021.06.20.449180.

Tseng YW, Diedrichsen J, Krakauer JW, Shadmehr R, Bastian AJ (2007) Sensory prediction errors drive cerebellum-dependent adaptation of reaching. $J$ Neurophysiol 98:54-62.

Van der Kooij K, Wijdenes LO, Rigterink T, Overvliet KE, Smeets JBJ (2018) Reward abundance interferes with error-based learning in a visuomotor adaptation task. PLoS One 13.

Wagenmakers EJ, Brown S (2007) On the linear relation between the mean and the standard deviation of a response time distribution. Psych Review 114:830-841.

Wang J, Bao S, Tays GD (2019) Lack of generalization between explicit and implicit visuomotor learning. PLoS One 14:e0224099.

Wei K, Körding K (2009) Relevance of error: What drives motor adaptation? J Neurophysiol 101:655-664.

Yin C, Wei K (2020) Savings in sensorimotor adaptation without an explicit strategy. J Neurophysiol 123:1180-1192.

Yin $\mathrm{H}$, Knowlton BJ (2006) The role of the basal ganglia in habit formation. Nat Rev Neurosci 7:464-476.

Yin HH, Ostlund SB, Knowlton BJ, Balleine BW (2005) The role of the dorsomedial striatum in instrumental conditioning. Eur J Neurosci 22:513-523. 


\section{FIGURE 1}

A

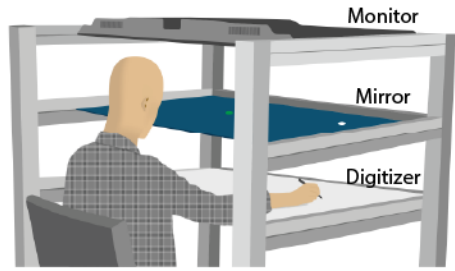

B

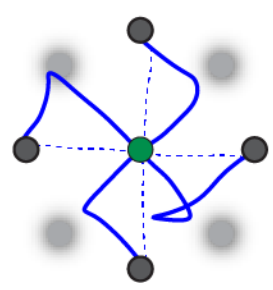

C

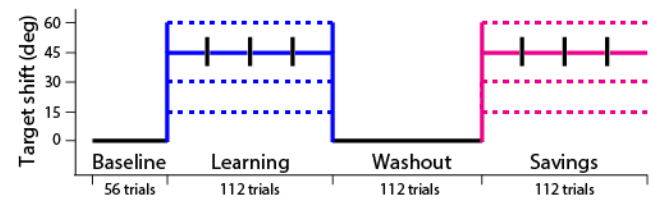

D

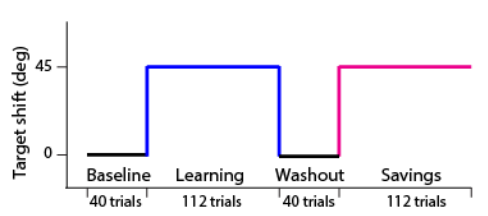

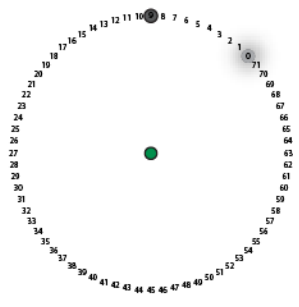

984

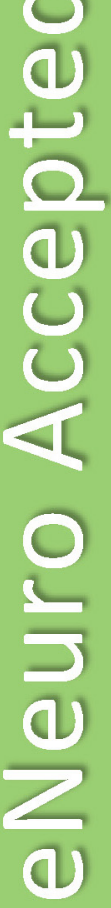


FIGURE 2
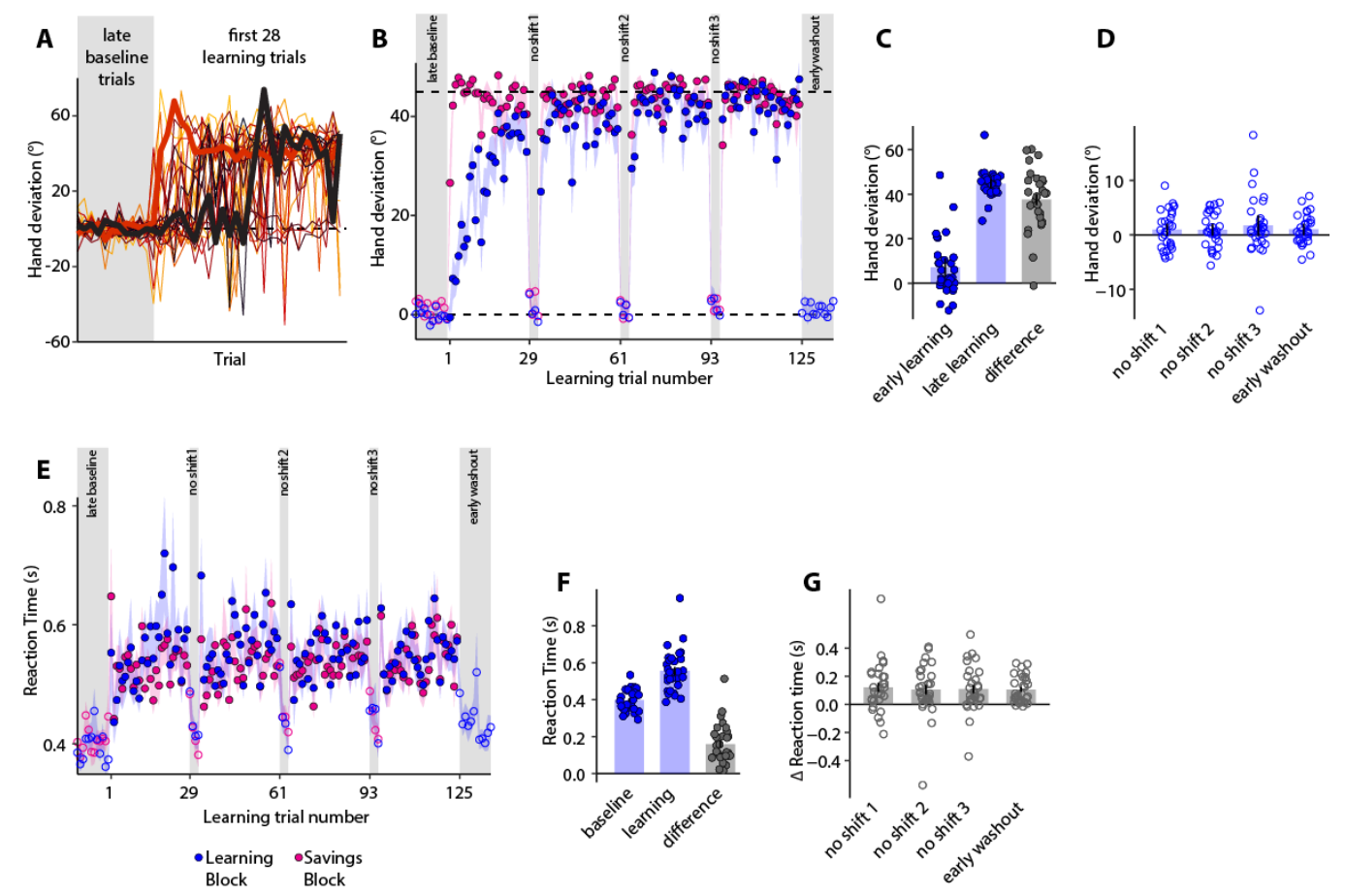

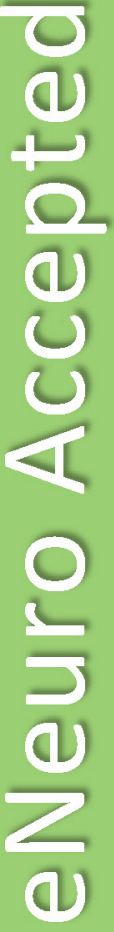

985

光 


\section{FIGURE 3}
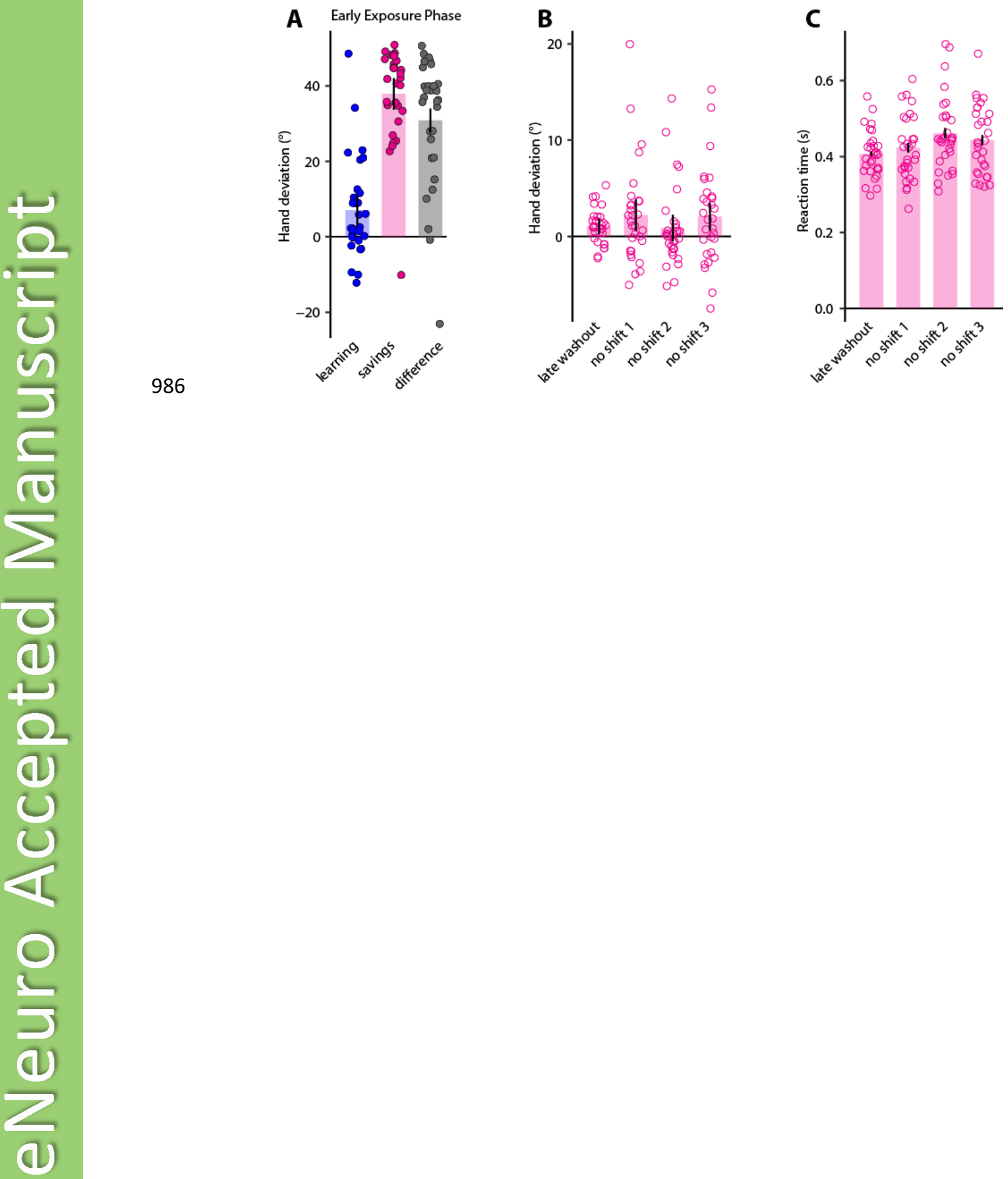


\section{FIGURE 4}

A

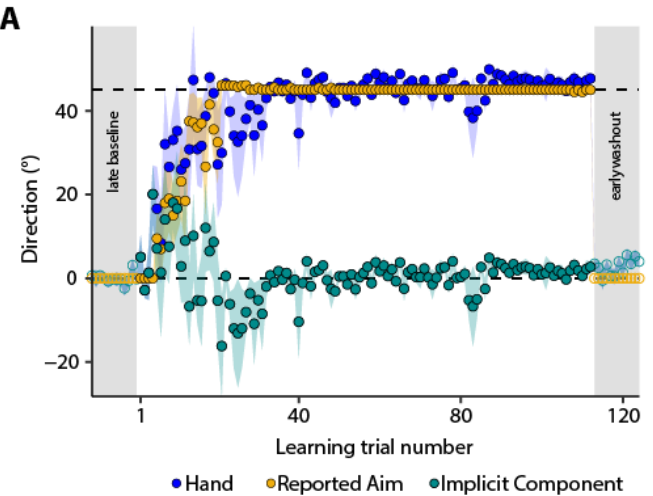

C

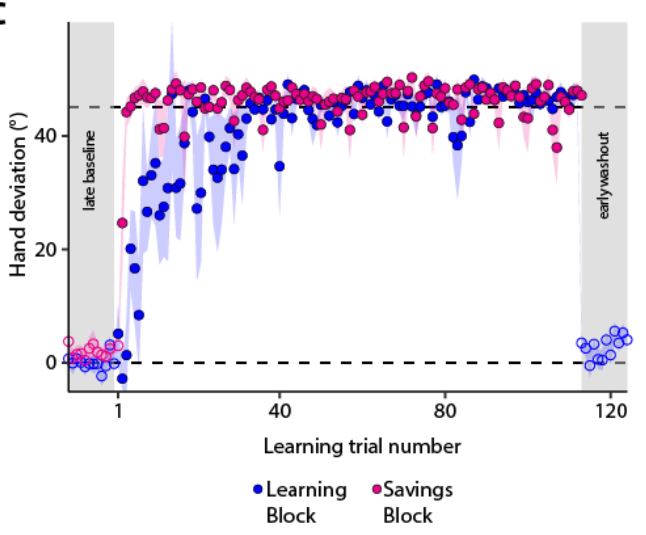

B

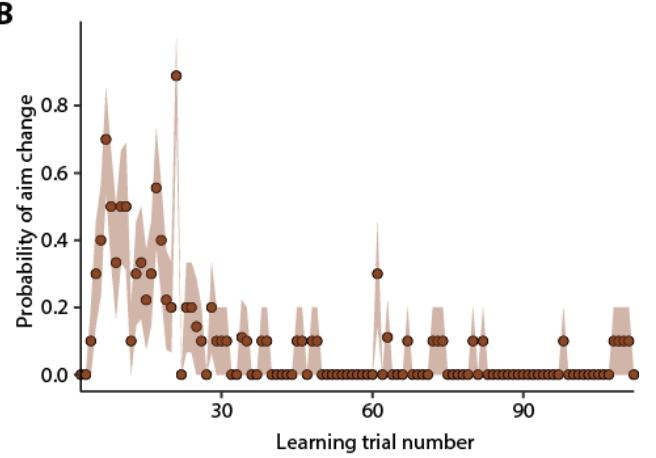

D Early Exposure Phase

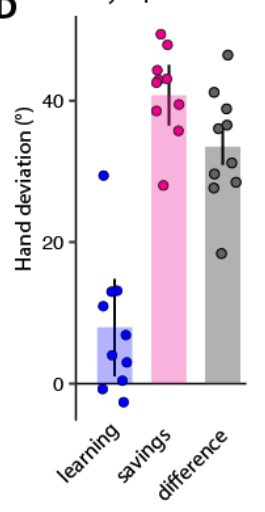


FIGURE 5

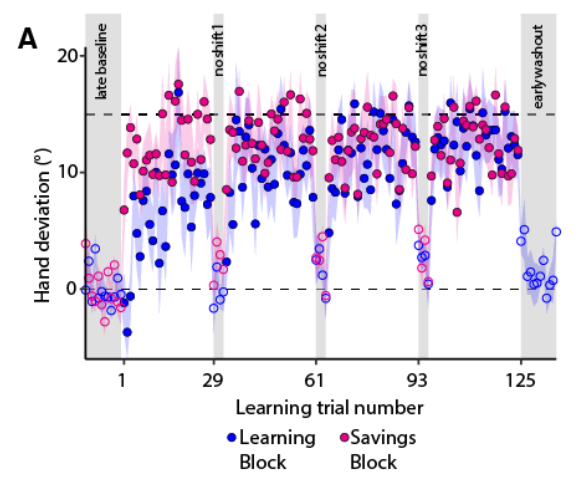

$15^{\circ}$ shift

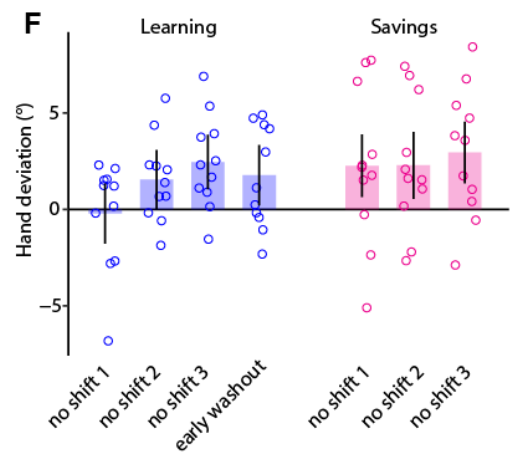

$30^{\circ}$ shift
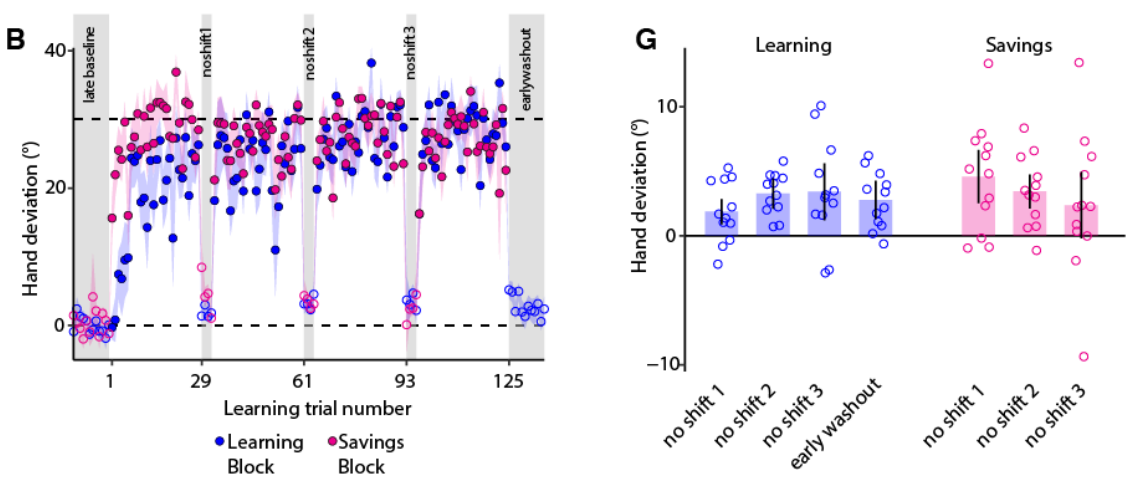

$60^{\circ}$ shift
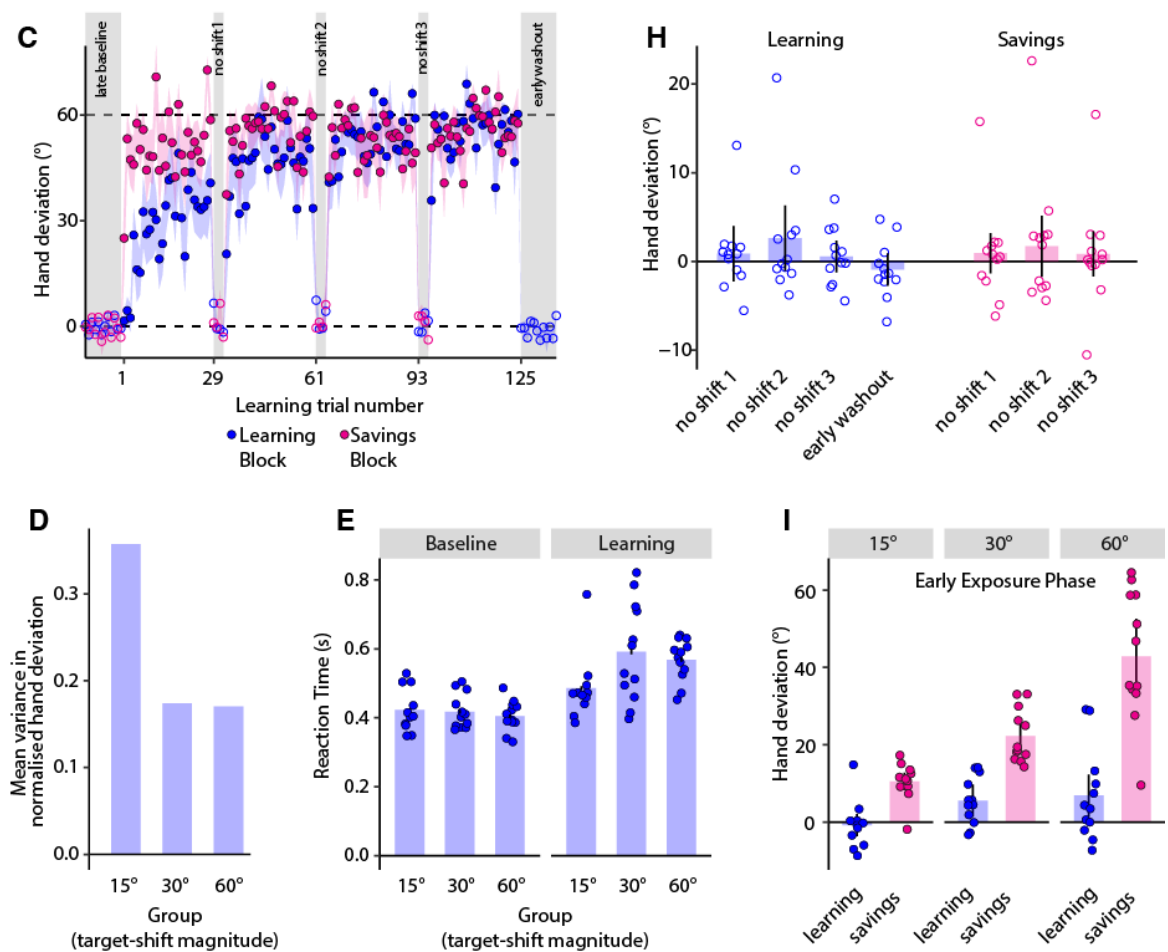
늘

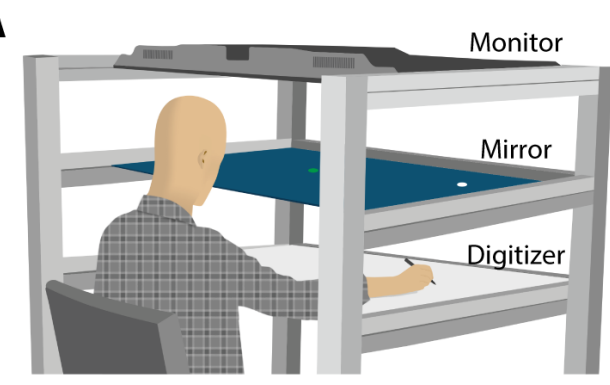

(0)

(d) $\mathrm{c}$

는

0

(d)

(U)

(v)

$\checkmark 5$

(2)

늑

(a)

(d)
B

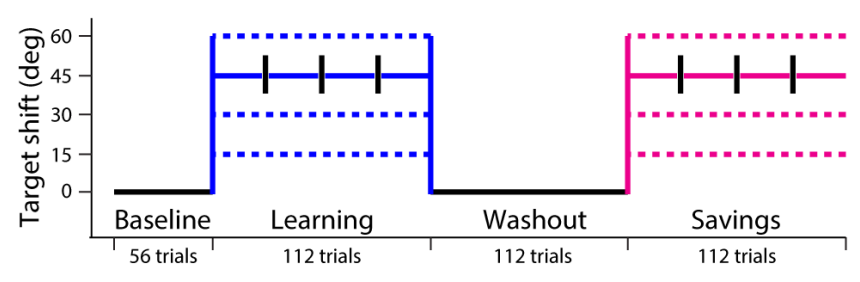

D
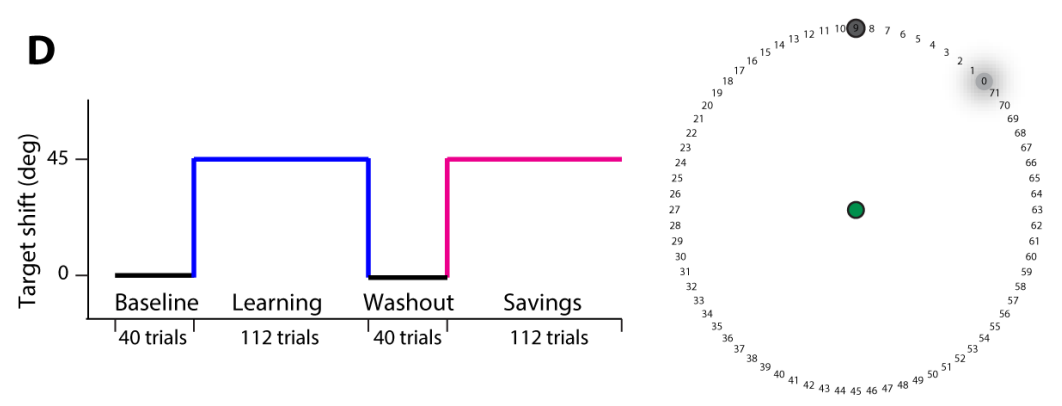

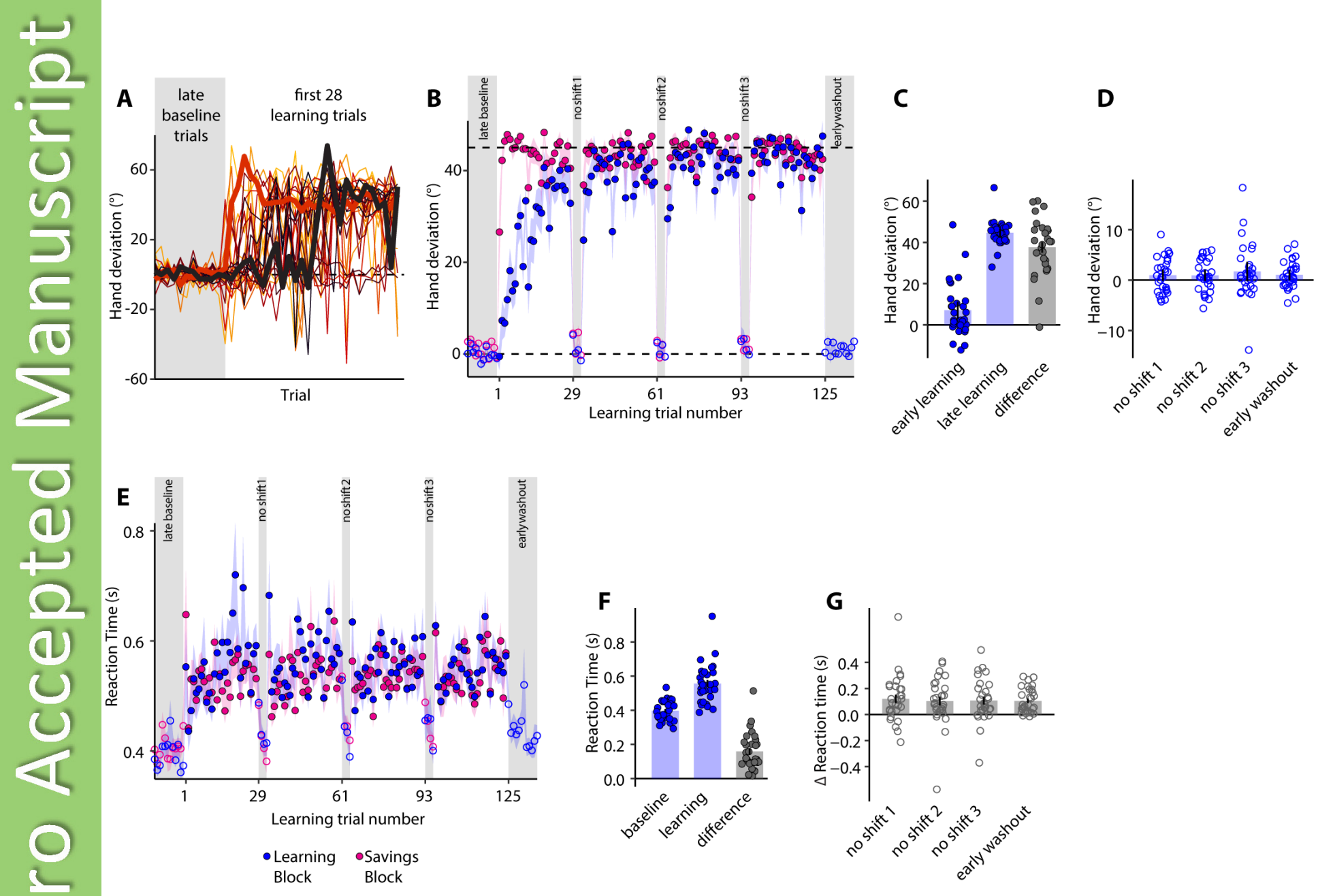

(d) 

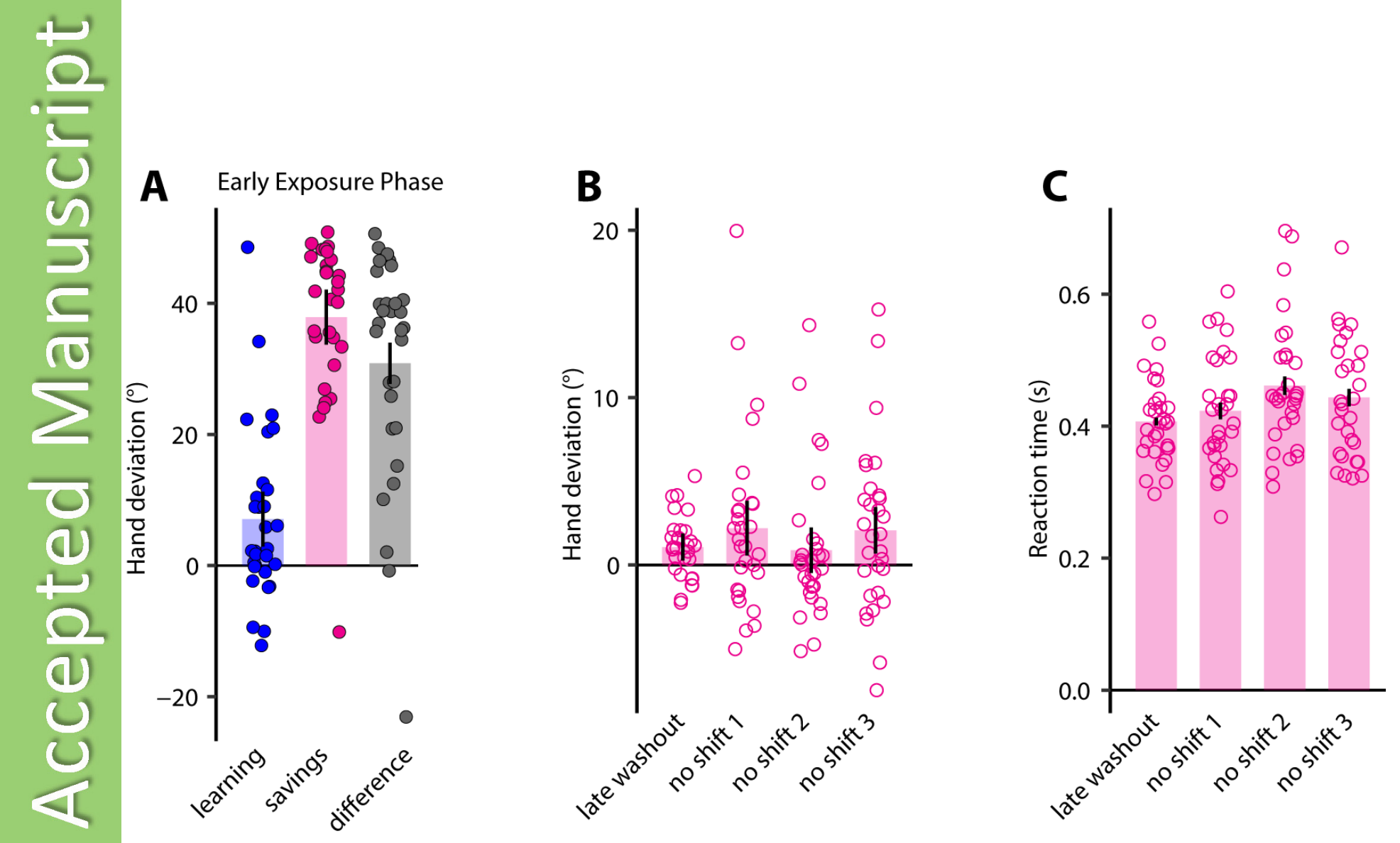

(

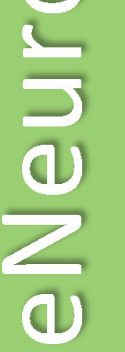



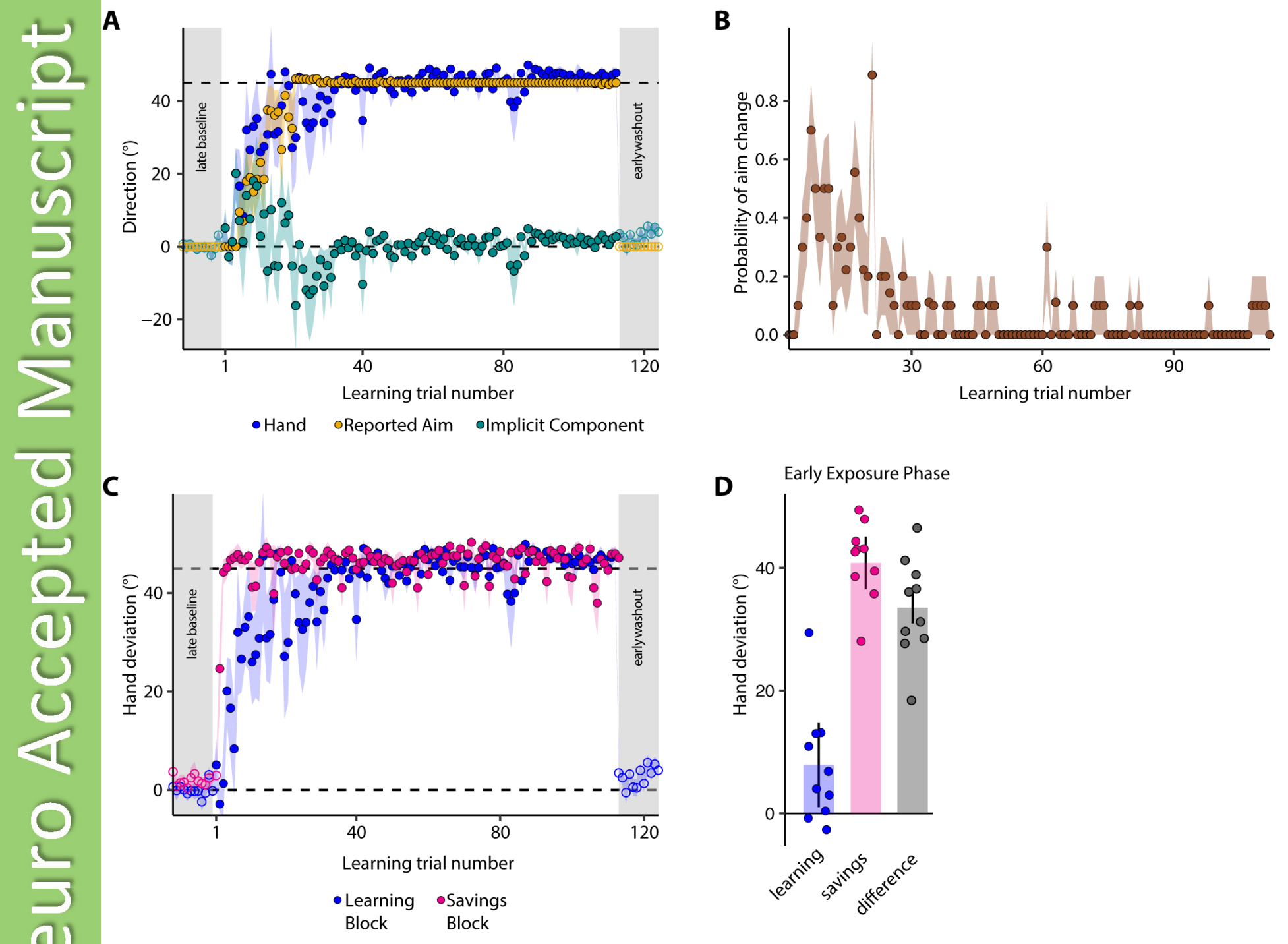
$15^{\circ}$ shift
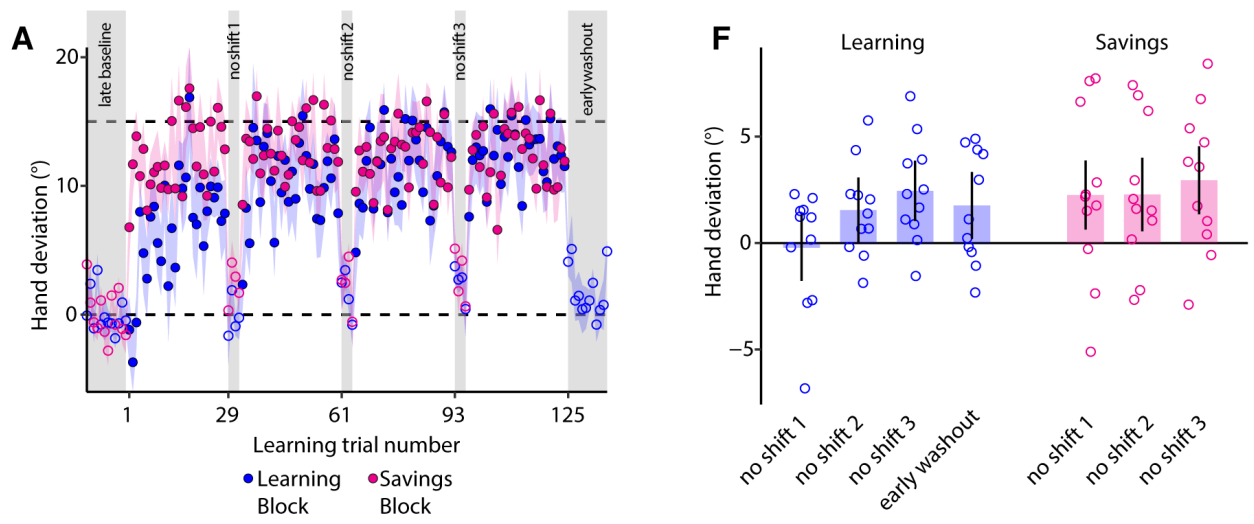

0 Block Block

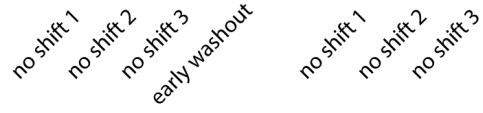

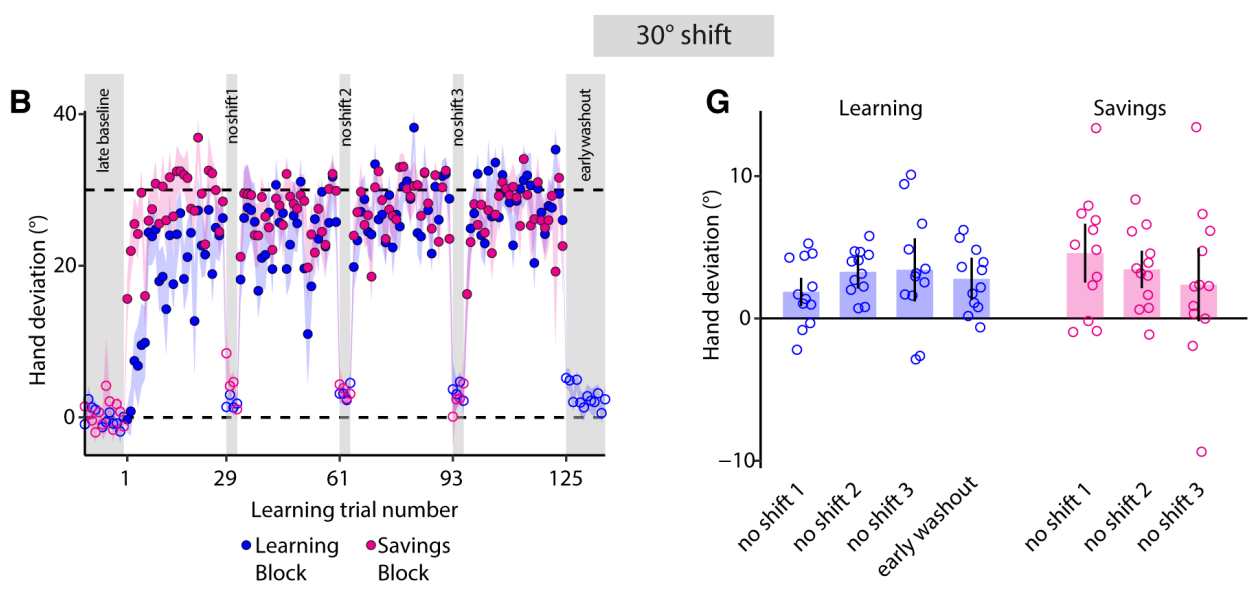

(d)

$60^{\circ}$ shift
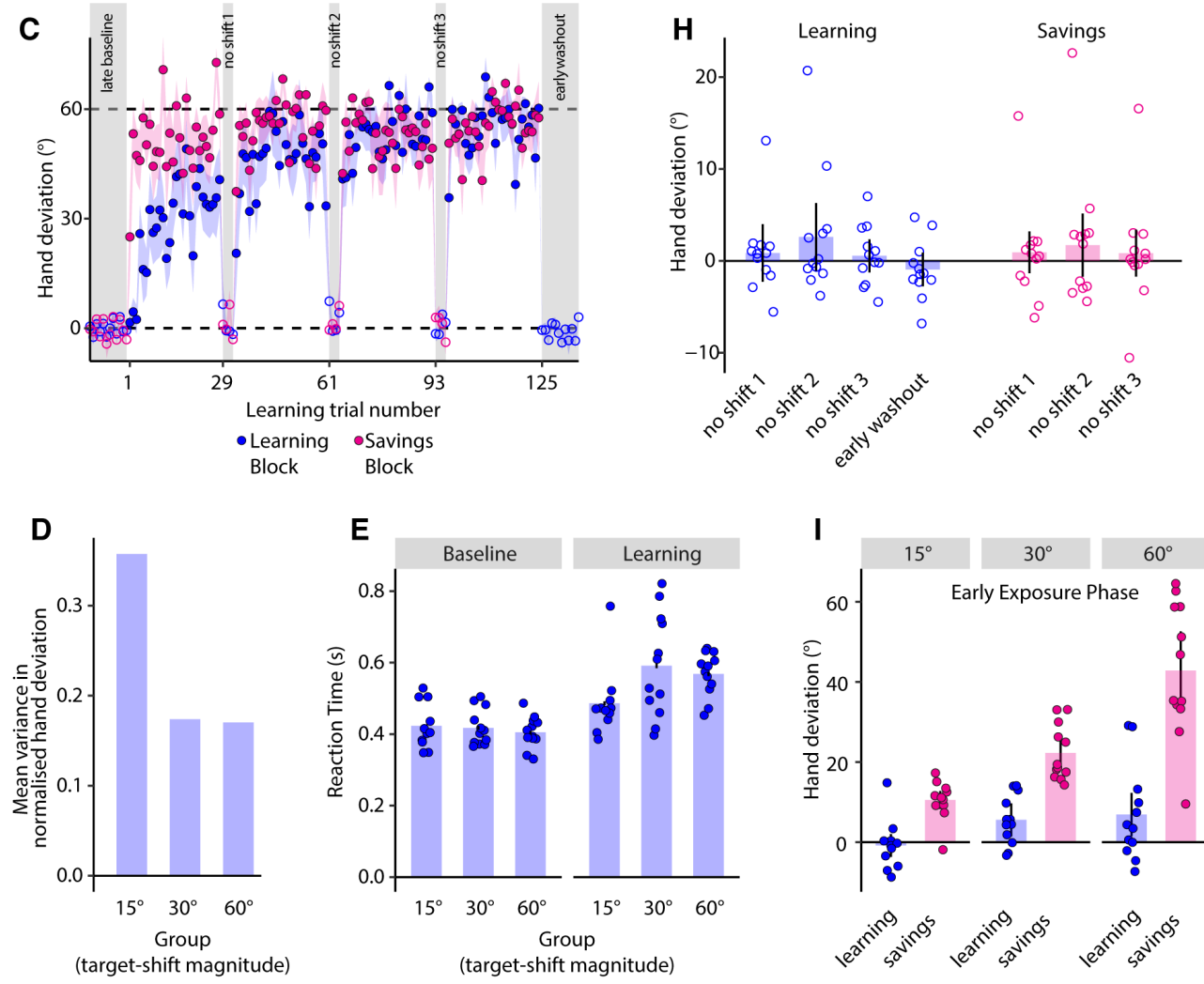